\title{
Adenovirus-mediated HIF-1 $\alpha$ gene transfer promotes repair of mouse airway allograft microvasculature and attenuates chronic rejection
}

\author{
Xinguo Jiang,, ${ }^{1}$ Mohammad A. Khan, ${ }^{1}$ Wen Tian, ${ }^{1}$ Joshua Beilke, ${ }^{2}$ Ramesh Natarajan, ${ }^{3}$ \\ Jon Kosek, ${ }^{4}$ Mervin C. Yoder, ${ }^{5}$ Gregg L. Semenza, ${ }^{6}$ and Mark R. Nicolls ${ }^{1}$

\begin{abstract}
1Department of Medicine, VA Palo Alto Health Care System/Stanford University School of Medicine, Stanford, California, USA. ${ }^{2}$ Novo Nordisk, Seattle, Washington, USA. ${ }^{3}$ Department of Medicine, Virginia Commonwealth University, Richmond, Virginia, USA. ${ }^{4}$ Department of Pathology, VA Palo Alto Health Care System/Stanford University School of Medicine, Stanford, California, USA. ${ }^{5}$ Department of Biochemistry and Molecular Biology, Indiana University School of Medicine, Indianapolis, Indiana, USA. ${ }^{6}$ Vascular Program, Institute for Cell Engineering, Departments of Pediatrics, Medicine, Oncology, Radiation Oncology, and Biological Chemistry, and McKusick-Nathans Institute of Genetic Medicine, Johns Hopkins University School of Medicine, Baltimore, Maryland, USA.
\end{abstract}

\begin{abstract}
Chronic rejection, manifested as small airway fibrosis (obliterative bronchiolitis [OB]), is the main obstacle to long-term survival in lung transplantation. Recent studies demonstrate that the airways involved in a lung transplant are relatively hypoxic at baseline and that $O B$ pathogenesis may be linked to ischemia induced by a transient loss of airway microvasculature. Here, we show that HIF-1 $\alpha$ mediates airway microvascular repair in a model of orthotopic tracheal transplantation. Grafts with a conditional knockout of Hifla demonstrated diminished recruitment of recipient-derived $\mathrm{Tie}^{+}$angiogenic cells to the allograft, impaired repair of damaged microvasculature, accelerated loss of microvascular perfusion, and hastened denudation of epithelial cells. In contrast, graft HIF-1 $\alpha$ overexpression induced via an adenoviral vector prolonged airway microvascular perfusion, preserved epithelial integrity, extended the time window for the graft to be rescued from chronic rejection, and attenuated airway fibrotic remodeling. HIF-1 $\alpha$ overexpression induced the expression of proangiogenic factors such as $S d f 1, P l g f$, and Vegf, and promoted the recruitment of vasoreparative Tie2 ${ }^{+}$cells. This study demonstrates that a therapy that enhances vascular integrity during acute rejection may promote graft health and prevent chronic rejection.
\end{abstract}

\section{Introduction}

The main limitation to long-term survival of a lung transplant is chronic rejection, which is manifested by a terminal airway fibrotic process that presents clinically as the bronchiolitis obliterans syndrome (BOS). The cumulative incidence of BOS within 6 years of transplantation is greater than $50 \%$ (1). Despite identification of risk factors such as acute cellular rejection, lymphocytic bronchitis/bronchiolitis, and CMV infection, the mechanisms by which BOS develops remain elusive $(2,3)$. Recent autopsy studies reveal a marked loss of microvasculature in the pre-obliterative bronchiolitis (pre-OB) foci of human lung transplants, which suggests that a loss of microcirculation and airway ischemia precede the onset of OB $(4,5)$. Clinical studies from other solid organ transplants, such as liver and kidney, also demonstrate that chronic rejection develops after a loss of functional microvasculature $(6,7)$. In a preclinical model of lung transplantation, we have shown that without immunosuppression, acute rejection eventually results in rejection of the donor microvasculature and a complete cessation of blood flow to the transplant. In this model, the presence of a functional microvasculature is essential for airway allografts to be rescued with immunosuppression from chronic rejection (8). These clinical and preclinical findings cumulatively suggest that loss of the microvascular circulation may be a fundamental cause of chronic

Conflict of interest: The authors have declared that no conflict of interest exists. Citation for this article: J Clin Invest. 2011;121(6):2336-2349. doi:10.1172/JCI46192. rejection. However, little is known about how microvessels are altered by rejection and whether manipulation of this process can change the final outcomes of acute rejection episodes.

Ischemia is the principal stimulus that induces neovascularization (9). Expression of virtually all proangiogenic growth factors is induced by hypoxia through the transcriptional activity of HIF-1 (10). HIF-1 is a heterodimer composed of a constitutively expressed HIF-1 $\beta$ subunit and an oxygen-regulated HIF- $1 \alpha$ subunit (11). AdCA5, an adenovirus vector encoding a constitutively active form of HIF- $1 \alpha$, has been demonstrated in several animal models to promote angiogenesis and accelerate recovery from tissue ischemia (12-14). HIF-1-mediated transcriptional responses orchestrate the expression of proangiogenic growth factors that facilitate angiogenesis by directly activating resident endothelial cells as well as recruiting circulating angiogenic cells $(14,15)$. Tie2 is a promoter that has been used to identify several subpopulations of cells that participate in neovascularization (16-19). Mice expressing the GFP or LacZ transgene under the control of Tie2 have proven useful in tracking the fate of heterogeneous and migrating cell populations (e.g., CD45-CD $31^{+}$endothelial cells, Tie2-expressing monocytes [TEMs]), which participate in new vessel formation (19-21).

Several animal models, including orthotopic tracheal transplant (OTT), heterotopic tracheal transplant, and orthotopic lung transplant, have been used to study the pathology associated with human lung transplantation. To date, rodent models have not 


\section{A Normal trachea \\ A Normal trachea}

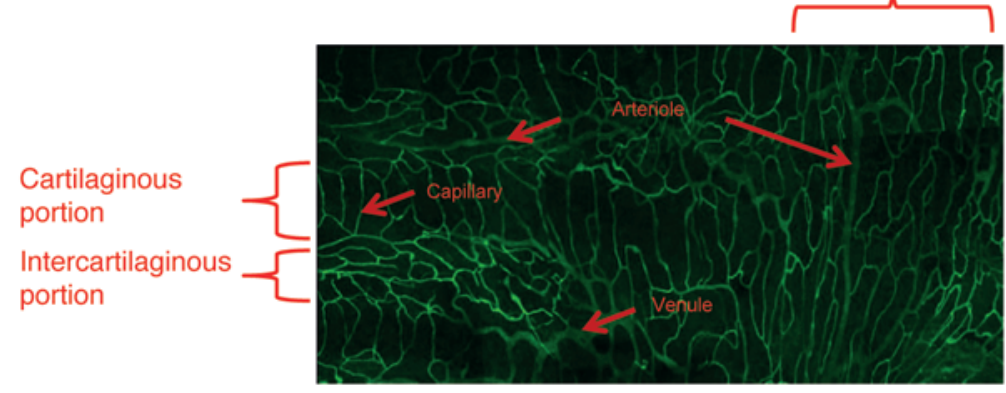

B Chronically rejected trachea allograft

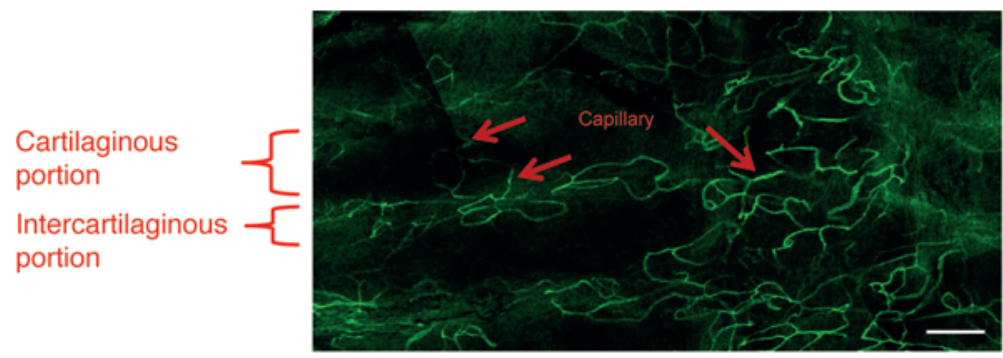

\section{Figure 1}

Remodeling of tracheal microvasculature in chronic rejection following transplantation. (A) Normal trachea. Arterioles and venules are located between cartilage rings and in the noncartilaginous (anatomically posterior) membranous portion. Capillaries are located overlying the cartilaginous portions. (B) Day-56 chronically rejected allograft. Only capillaries are evident in the different portions of the trachea. Fewer vessels are seen in the cartilaginous portion, and the microvasculature becomes tortuous and disorganized in the intercartilaginous and the membranous portions. Both panels are composited of separate images. Scale bar: $100 \mu \mathrm{m}$.

convincingly replicated OB lesions. However, although OTT does not develop the clinically relevant airway obliteration, it reproducibly develops subepithelial fibrosis and mimics the clinically relevant pathology of lymphocytic bronchitis, a large airway correlate of BOS (22). Moreover, the OTT model is an ideal system to study airway microvascular repair and remodeling that occurs during alloimmune injury because of the well-organized planar anatomy of airway microvasculature. OTT accurately models transplantation-associated changes in the large airways, an area of tissue that is temporarily devitalized because the bronchial artery circulation (which conducts blood with high $\mathrm{pO}_{2}$ from the aorta) is not usually surgically restored at the time of transplantation. We have previously shown that OTTs undergoing acute rejection are relatively hypoxic compared with nonrejecting tracheal tissue and undergo sequential damage characterized first by microvascular injury, followed by airway ischemia, and finally, reperfusion with active neovascularization (8). Our recent clinical study revealed that human lung transplant airways also are relatively hypoxic at baseline compared with both native (diseased) and control airways (23). These recent findings suggest that, during rejection, increased hypoxia and ischemia may trigger an adaptive response to promote neovascularization of the allograft through activation of HIF- $1 \alpha$. HIF- $1 \alpha$ consequently may be one of the central factors that help to maintain a functional microvasculature in transplanted organs.
In this study, we carefully characterized the timedependent microvascular changes that occur during allograft rejection, focusing on how the donor and recipient tissues interact with each other to facilitate vascular recovery. We also examined the normal role that Hifla plays in the repair of the damaged microvasculature of an OTT. The main objective for these experiments was to determine whether limiting airway hypoxia and ischemia, through enhanced maintenance or accelerated recovery of the airway microvasculature, could prevent chronic rejection.

\section{Results}

Revascularization of chronically rejected OTTs is incomplete, and remodeled vessels are disorganized. We previously demonstrated that, without immunosuppression, acute transplant rejection leads to the loss of a functional microcirculation, meaning that the small vessels supplying the rejecting airway allografts are destroyed and stop perfusing. Once the microvasculature is lost, late administration of immunosuppression can no longer rescue the airway from developing chronic rejection (8). In these OTT studies, acute rejection is simply defined as the inflammatory phase that progresses to chronic rejection in the absence of early intervention with immunosuppression. Chronic rejection, which follows untreated acute rejection, is characterized by relatively less inflammation, increasing subepithelial fibrosis, and the development of a flattened, dysplastic epithelium $(8,24)$. In our prior study (8), we further demonstrated that after prolonged ischemia, which begins on day 10 , the airways eventually become neovascularized and that by day 28 , there is evidence of partial restoration of blood flow. This process involves a reinvestment of blood vessels into now chronically rejected airways. Similar results have been reported in lung transplant autopsy studies $(4,5)$.

For the current study, we hypothesized that preserving a functional microvasculature, either by delaying its loss (by promoting donor microvascular integrity) or by accelerating its recovery (through increased growth of recipient vessels into donor airways), would delay or prevent the onset of chronic rejection. To understand how the damaged donor microvasculature is normally repaired and how alloimmune injury shapes microvascular remodeling, we compared the microvasculature of a chronically rejected tracheal allograft with that of a normal trachea. Normal microvasculature is highly organized with subepithelially arranged arterioles, venules, and capillaries (Figure 1A). In contrast, revascularization of chronically rejected airways is incomplete, and the remodeled vessels are morphologically distinct from normal microvasculature: capillaries in the cartilaginous portion are scarce and no arterioles or venules are found in either the intercartilaginous or membranous portion (Figure 1B). Moreover, remodeled vessels in chronically rejected airways are tortuous, disorganized (Figure 1B), and resemble immature and unstable tumor microvasculature (25).

Coverage of endothelial cells by pericytes results in vessels with a more mature and stable phenotype; unstable tumor microvessels often lack this pericyte association (26). We therefore examined the endothelial coverage by pericytes in tracheal capillaries. Double 
A

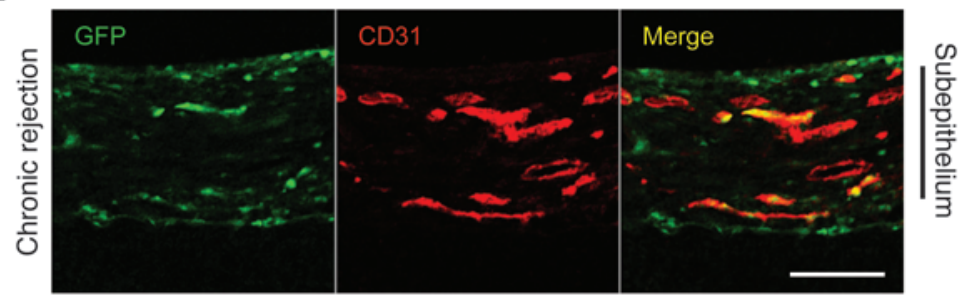

C

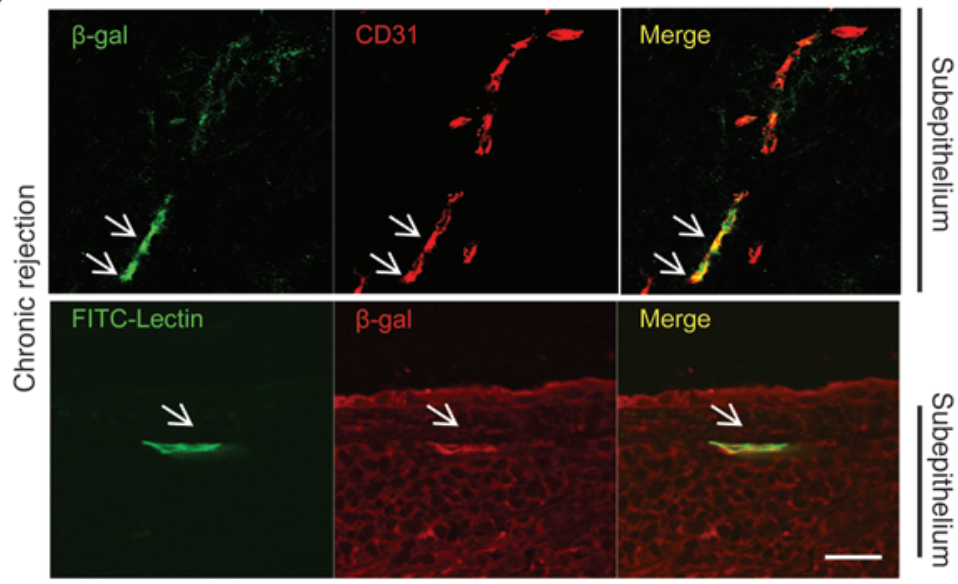

$\mathbf{F}$

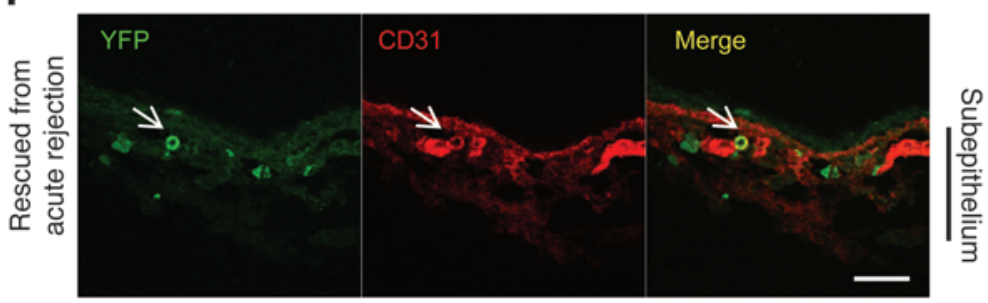

B
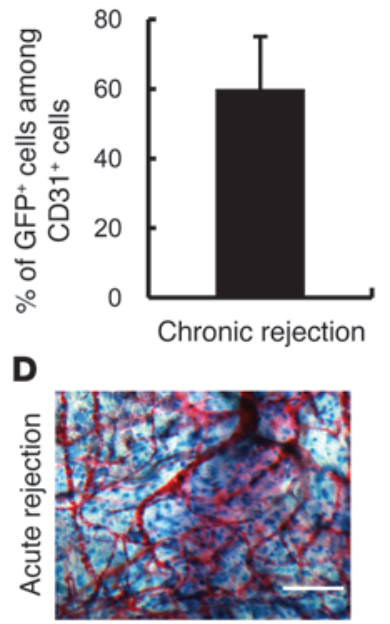

Whole mount

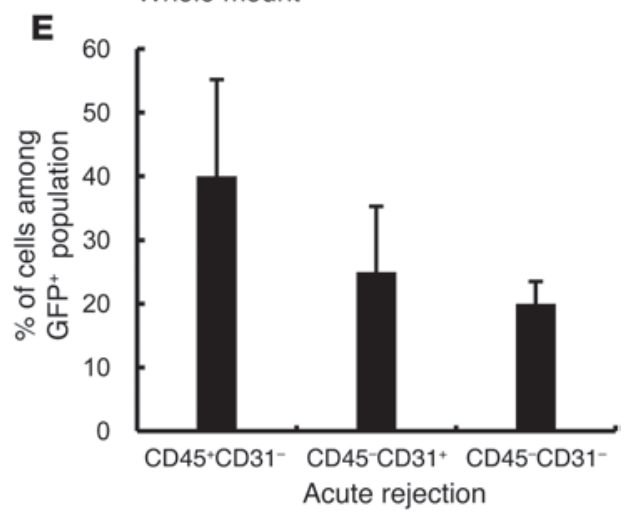

Figure 2

Recipient-derived Tie2 ${ }^{+}$cells contribute to microvascular remodeling in chronic rejection and to repair of donor vessels rescued from acute rejection by immunosuppression. (A) Immunofluorescence (IF) staining of a cross section (with subepithelium marked by the black solid line on the right side of the images here and in the following figures) of day-56 allografts (Balb/c to FVB [actin-EGFP]) shows that the majority of CD31+ cells (red) are GFP+ (green) and of recipient origin. (B) The percentage of GFP+CD31+ cells among CD31+ cells was calculated as the percentage of the recipient contribution to the remodeled microvasculature $(n=6)$. (C) Chronically rejecting day-28 allografts (Balb/c to FVB[Tie2-LacZ]) shows that $\beta$-gal ${ }^{+}$(arrows; green) cells form tube-like structures and become part of CD31+ (arrows; red) microvasculature (top panel). Perfusion coupled with IF staining analysis shows that $\beta$-gal ${ }^{+}$(arrow; red) cells are perfused by FITC-conjugated lectin (arrow; green) (lower panel). (D) Recipient mice bearing acutely rejecting day-6 allografts (Balb/c to FVB[Tie2-LacZ]) were perfused with red India ink, and then whole-mount trachea allografts were prepared following X-gal staining. Recipient-derived Tie2 ${ }^{+}$cells (blue) are scattered in the allograft and mainly outside of the vessels (red). (E) Recipient-derived GFP+ ${ }^{+}$cells of day- 6 allografts (Balb/c to FVB[Tie2-EGFP]) were analyzed for CD45 and CD31 expression by flow cytometry. (F) Balb/c tracheas were first transplanted into B6 (with double transgene: Tie2-Cre, ROSA26EYFP) mice, and then the day-6 grafts were retransplanted into WT B6 mice subjected to immunosuppression; retransplanted grafts were harvested 28 days later. Grafts are histologically normal. IF staining shows that some YFP+ cells (arrow; green) become part of CD31+ (arrow; red) vasculature. Scale bars: $100 \mu \mathrm{m}$ $(\mathbf{A}$ and $\mathbf{D}) ; 20 \mu \mathrm{m}$ (C and F). Data are shown as mean \pm SEM.

staining for the endothelial marker CD31 with the mural marker $\mathrm{NG} 2$ or $\alpha$-SMA (which label pericytes) revealed that in rejecting allografts there were fewer pericyte-covered vessels and that these vessels had smaller lumens (Supplemental Figure 1, B and C; supplemental material available online with this article; doi:10.1172/ JCI46192DS1). These data suggest that remodeled microvessels of chronically rejected airways are relatively immature and unstable.

Recipient-derived Tie $2^{+}$cells are recruited to the allograft and participate in the repair of graft microvasculature in both acute and chronic rejection. Clinical studies with kidney transplants suggest that replacement of donor endothelial cells by recipient-derived cells not only indicates endothelial injury but also suggests that recipient repair of injured donor microvasculature is possible $(27,28)$. To determine whether replacement of donor endothelial cells by recipient cells occurs in airway transplants undergoing chronic rejection, actin-EGFP transgenic mice (EGFP expressed under the control of the actin promoter) were used as recipients. After 56 days of rejection, the majority $(62.2 \% \pm 18.5 \%)$ of CD $31^{+}$ endothelial cells are $\mathrm{GFP}^{+}$and thus of recipient origin (Figure 2, $\mathrm{A}$ and $\mathrm{B})$. These data indicate that recipient-derived cells prominently contribute to the remodeling of the microvasculature of OTTs undergoing chronic rejection. 
A

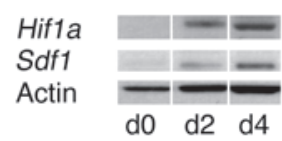

B
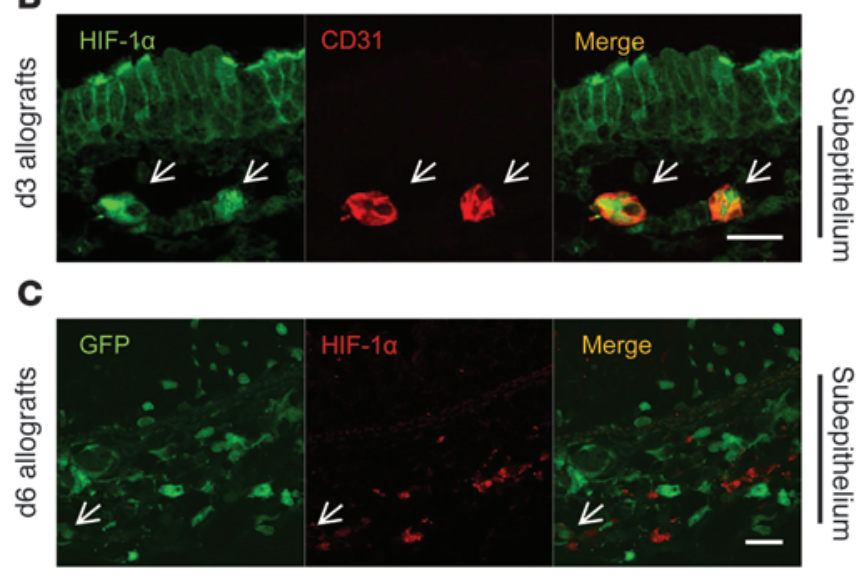

D

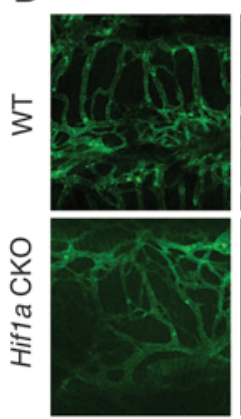

d4

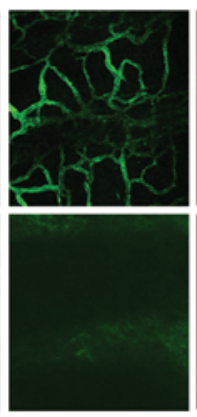

d8

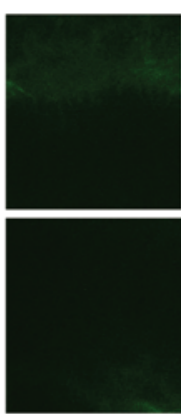

d10

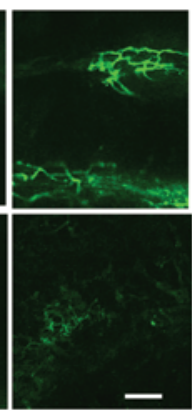

d21
Acute rejection
E

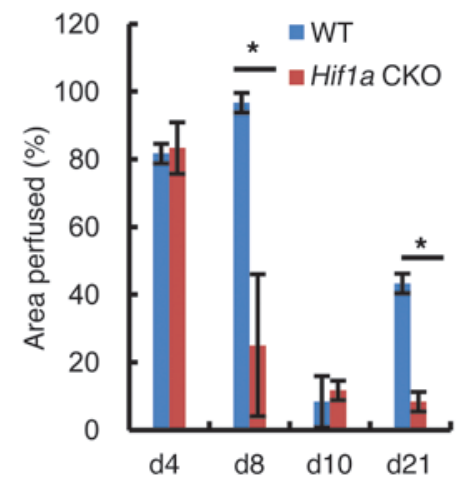

G
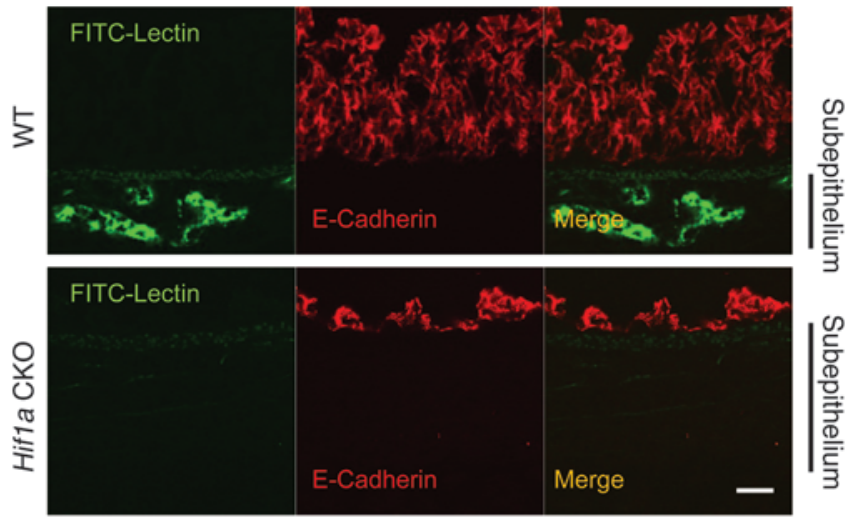

d8 allografts

H

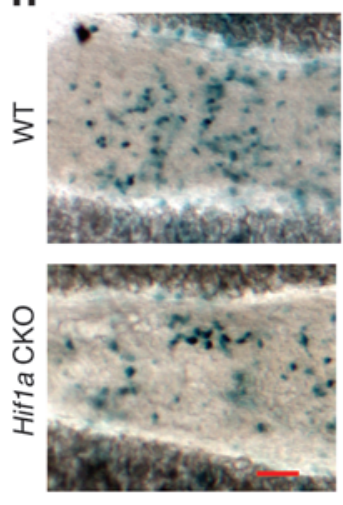

Whole mount
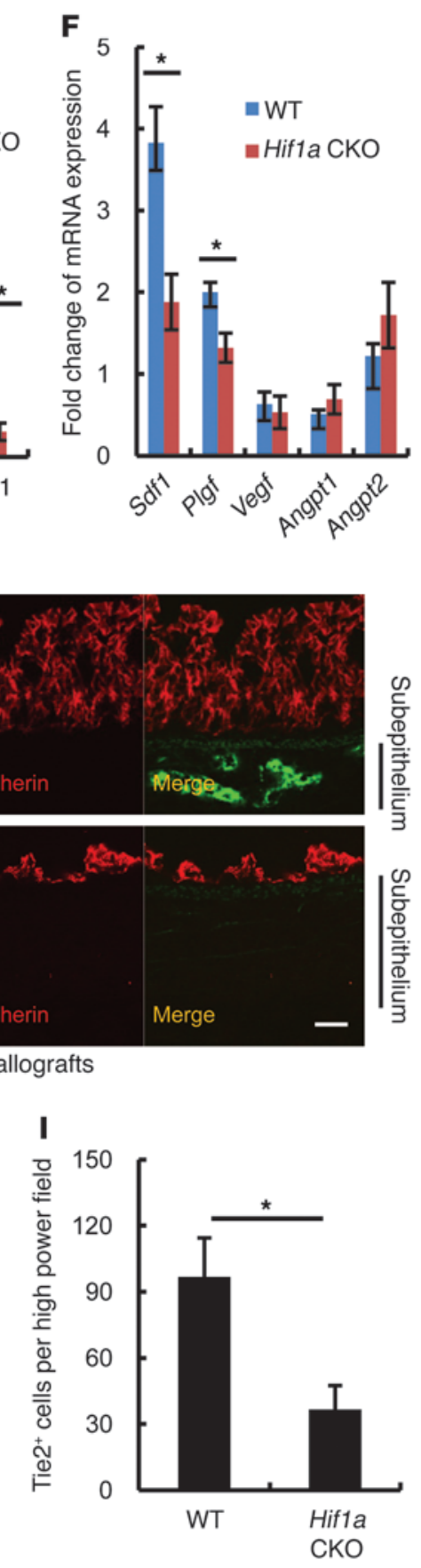

Figure 3

Hif1a is essential for early microvascular repair of trachea allografts. (A) Western blotting of Hif1a and Sdf1 in day-2 and day-4 trachea allografts. Actin was used as an internal control. The lanes were run on the same gel but were noncontiguous. (B) IF staining of day-3 allografts (Balb/c to B6) shows that Hif1a+ cells (arrows; green) express CD31 (arrows; red). (C) IF staining of day-6 allografts (Balb/C to FVB[actin-EGFP]) shows that only a small percentage of Hif1 $\mathrm{a}^{+}$cells (arrow; red) are of recipient origin (arrow; green). (D) Microvascular perfusion of WT trachea allografts is completely lost at day 10 , followed by partial revascularization at day 21 . However, complete loss of vascular perfusion of Hif1a CKO trachea starts 2 days earlier (day 8), followed by minimal revascularization by day 21. (E) Percentage of perfusion area of trachea allograft was calculated $\left(n=4-6\right.$; $\left.{ }^{*} P<0.05\right)$. (F) Real-time RT-PCR analysis of angiogenic factors of day-6 WT and Hif1a CKO allografts $\left(n=4\right.$; $\left.{ }^{*} P<0.05\right)$. (G) FITCconjugated lectin perfusion coupled with IF staining of day-8 WT allografts shows normal microvascular perfusion (green) and pseudostratified columnar epithelium (E-cadherin staining; red) (top panel). However, in the acutely rejecting Hif1a CKO allograft, there is a loss of perfusion in the subepithelial area, and the epithelium is flat with some basement membrane exposed (lower panel). (H) Day-6 Hif1a CKO or WT allografts demonstrated that fewer Tie2+ cells (blue) were recruited to Hif1a CKO allograft. (I) Tie2 ${ }^{+}$cells per high power field were calculated to confirm the result shown in $\mathbf{H}\left(n=5 ;{ }^{*} P<0.05\right)$. Scale bars: $20 \mu \mathrm{m}$ (B, C, and $\left.\mathbf{F}\right) ; 100 \mu \mathrm{m}$ (D and $\left.\mathbf{G}\right)$. Data are shown as mean $\pm \mathrm{SEM}$. 
Tie2 has been used to identify cells that participate in neovascularization of tumors and ischemic tissues (16-19). However, its role in airway microvascular repair and remodeling has not been studied. To examine whether recipient-derived Tie $2^{+}$cells contribute to airway revascularization, we transplanted $\mathrm{MHC}$-incompatible tracheas from $\mathrm{Balb} / \mathrm{c}$ donors into FVB recipients with a Tie2-LacZ transgene. We examined chronically rejected allografts and found that $\beta$-gal ${ }^{+}$ cells formed tube-like structures and expressed CD31 (Figure 2C). Further functional study showed that these $\beta$-gal ${ }^{+}$cells are able to be perfused by FITC-conjugated lectin (Figure 2C). Flow cytometry analysis showed that a significant fraction $(50.5 \% \pm 13.1 \%)$ of $\mathrm{CD} 1^{+}$cells are recipient-derived Tie $2^{+}$cells. These data collectively demonstrate that recipient-derived $\mathrm{Tie}^{+}$cells are a major source of endothelial cells of the reestablished vessels in chronic rejection and that these recipient-derived vessels are functional.

Because chronically rejected allografts that bear remodeled microvasculature following prolonged injury are already fibrotic and not rescuable with immunosuppression (8), we next sought to determine whether recipient-derived Tie $2^{+}$cells could also contribute to microvascular repair in acutely rejecting OTTs when the donor vasculature was still functional and airways were still capable of being restored to normal architecture (i.e., no subepithelial fibrosis, absent inflammation, and columnar pseudostratified epithelium). To determine whether recipient-derived Tie $2^{+}$cells were involved in early vascular repair in acutely rejecting OTTs, we assessed recruitment of recipient Tie $2^{+}$cells into day- 6 allografts. India ink perfusion together with X-gal staining showed a significant infiltration of recipient Tie $2^{+}$cells, which were scattered mainly outside of the perfusing donor-derived vessels (Figure 2D). Characterization of graft-infiltrating recipient $\mathrm{Tie} 2^{+}$cells by flow cytometry showed that the majority of the Tie $2^{+}$cells were $\mathrm{CD} 45^{+} \mathrm{CD} 31^{-}$, consistent with TEMs (16); other major populations were $\mathrm{CD}^{-} 5^{-} \mathrm{CD} 31^{+}$and $\mathrm{CD} 45^{-} \mathrm{CD} 31^{-}$cells (Figure $2 \mathrm{E}$ ), a result indicating that migrating $\mathrm{Tie} 2^{+}$cells represent a heterogeneous population of cells.

Next, we investigated whether these recipient Tie $2^{+}$cells, recruited during the early phase of acute rejection, incorporated into the donor vasculature. To facilitate this, we first established a lineage-tracing model by crossing ROSA26EYFP reporter mice with mice expressing Tie2-Cre (Supplemental Figure 2A). Immunohistochemistry showed that tracheal endothelial cells with double transgenes (Tie2-Cre and ROSA26EYFP) were $\mathrm{YFP}^{+}$, whereas nonendothelial cells were $\mathrm{YFP}^{-}$(Supplemental Figure 2B), suggesting that this strategy was able to faithfully and permanently mark the endothelial cells of the trachea. We used C57BL/6 (B6) mice with double transgenes (Tie2-Cre and ROSA26EYFP) as OTT recipients. Consistent with the Tie2-LacZ model, significant numbers of recipient $\mathrm{YFP}^{+}$cells were recruited to the donor airway 6 days following transplantation (data not shown). To determine whether these early recruited recipient-derived Tie $2^{+}$cells could be successfully incorporated into an injured donor vascular system, we retransplanted these day- $6 \mathrm{Balb} / \mathrm{c}$ allografts into naive second nontransgenic WT B6 recipients, which were treated with combined anti-LFA-1 and anti-CD40L monoclonal antibodies, a therapy that effectively reverses rejection in the OTT model (8). Because the second recipient does not express YFP, all $\mathrm{YFP}^{+}$cells expressed in the graft in the second recipient began as Tie $2^{+}$cells from the first recipient. This strategy permitted an examination of the fate of recipient $\mathrm{Tie} 2^{+}$cells recruited into donor airways during acute rejection. Twenty-eight days following retransplantation, the acute rejection was reversed, and the grafts were histologically normal. At this time, sporadic $\mathrm{YFP}^{+}$cells were found invested in the $\mathrm{CD} 31^{+}$donor microvasculature (Figure $2 \mathrm{~F}$ ), demonstrating that some early recruited recipient $\mathrm{Tie} 2^{+}$cells are able to become stable constituents of the donor microvasculature. Notably, extravascular Tie $2^{+}$cells (including TEMs) are largely absent in both grafts rescued from acute rejection and in chronically rejected airways (data not shown). Although it is likely that CD45-CD31+ Tie 2 cells were the principal recipient cells that incorporated into injured vessels, we cannot exclude the possibility that other Tie2 populations, such as $\mathrm{CD} 45^{+} \mathrm{CD} 31^{-}$TEMs and $\mathrm{CD} 45^{-} \mathrm{CD} 31^{-}$cells, may also have transformed to become donor endothelium.

In vitro studies have shown that Tie2 also identifies pericyte precursors with mesenchymal origin (16). To determine whether $\mathrm{Tie} 2^{+}$cells are able to differentiate into pericytes in alloimmuneinjured OTT, tracheas from Balb/c mice transplanted into B6 mice with double transgenes (Tie2-Cre and ROSA26EYFP) were evaluated for recipient contribution to donor pericyte coverage. Immunofluorescence staining analysis of chronically rejected allografts showed that some $\mathrm{YFP}^{+}$cells became Pdgfr $b^{+}$cells, which invest $\mathrm{YFP}^{+}$endothelial cells, suggesting these $P d g f r b^{+}$cells are part of the vasculature (Supplemental Figure 3A). Flow cytometry analysis confirmed the existence of YFP- and Pdgfrb-coexpressing cells, which accounted for about $6 \%-10 \%$ of total Pdgfr $b^{+}$pericytes (Supplemental Figure 3B). These data suggest that recipient-derived $\mathrm{Tie}^{+}$cells are also a source of the pericytes of the reestablished microvasculature in chronic rejection. Thus, recipient-derived Tie $2^{+}$cells materially participate in the microvascular repair in both acute and chronic rejection. With this understanding of the normal sequence of airway microvascular injury, loss, repair, and restoration, we next sought to investigate how graft-derived HIF-1 $\alpha$ influenced these processes.

HIF-1 $\alpha$ is essential for early tracheal allograft microvascular repair. HIF- $1 \alpha$ is the master regulator of the cellular response to ischemia and hypoxia (29). For example, HIF-1 $\alpha$ has been shown to be essential and sufficient for promoting neovascularization in mice with hind limb ischemia $(12,14)$. We therefore sought to determine whether HIF-1 $\alpha$ was important in the physiology of maintaining a viable airway circulation during rejection. We previously observed that hypoxia as well as active angiogenesis was present in OTTs during the acute rejection phase (8). We thus hypothesized that the repair of the alloimmune-injured airway microvasculature was HIF-1 $\alpha$ dependent. Tracheal Hif1 a protein was elevated at both 2 and 4 days following transplantation, and the expression of Sdf1, a chemokine involved in the recruitment of angiogenic cells to hypoxic areas, was also augmented (Figure 3A). Examination of day-3 allografts showed that $\mathrm{Hifla}^{+}$cells are principally CD $31^{+}$endothelial cells (Figure $3 \mathrm{~B}$ ), suggesting that endothelial cells primarily account for the increased Hifla expression in rejecting airways. Moreover, significant endothelial nuclear Hif1a was noted (Supplemental Figure 4A). Endothelial cells have been shown to be able to produce angiogenic growth factors under hypoxic conditions; histologic examination of Sdf1, Plgf, and Vegf proteins revealed that all of these proangiogenic factors are principally expressed by endothelial cells (Supplemental Figure 4, B-D). It has been shown that hypoxia also leads to augmented levels of HIF- $1 \alpha$ in cells of innate immunity $(30,31)$, and there is a notable and expected infiltration of recipient-derived immune cells to the donor airway during acute rejection (data not shown). We therefore assessed Hifla protein in recipient-derived immune cells. 
A
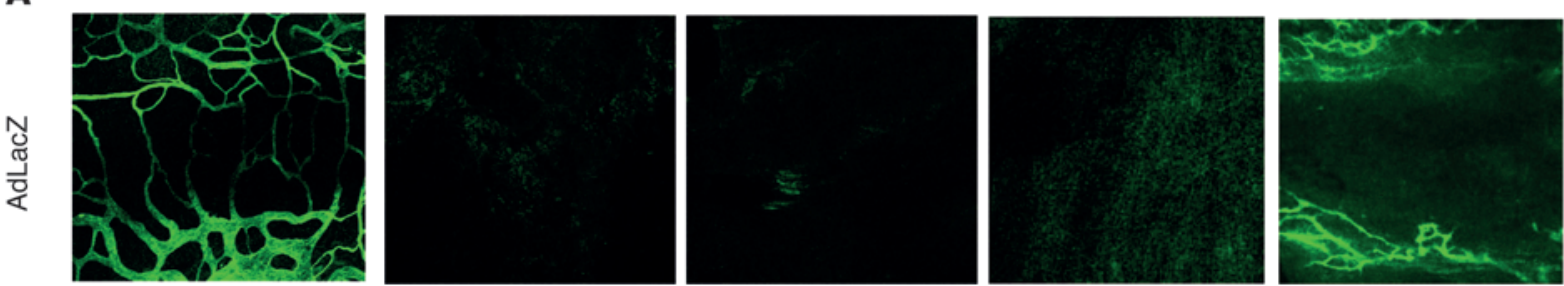

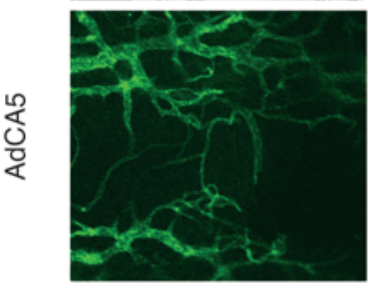

d8

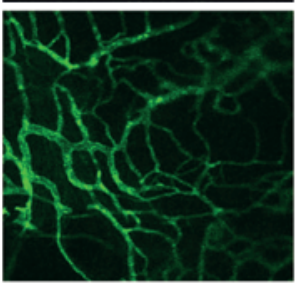

d10

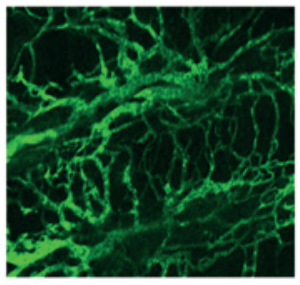

d12

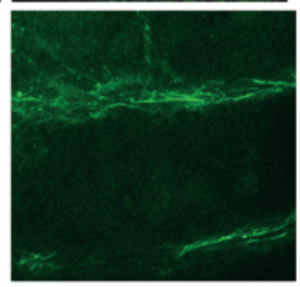

d14

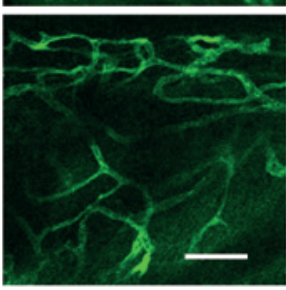

d21

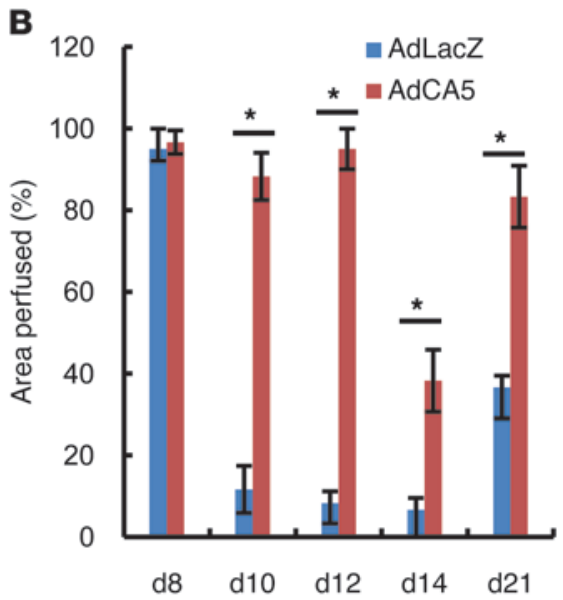

D
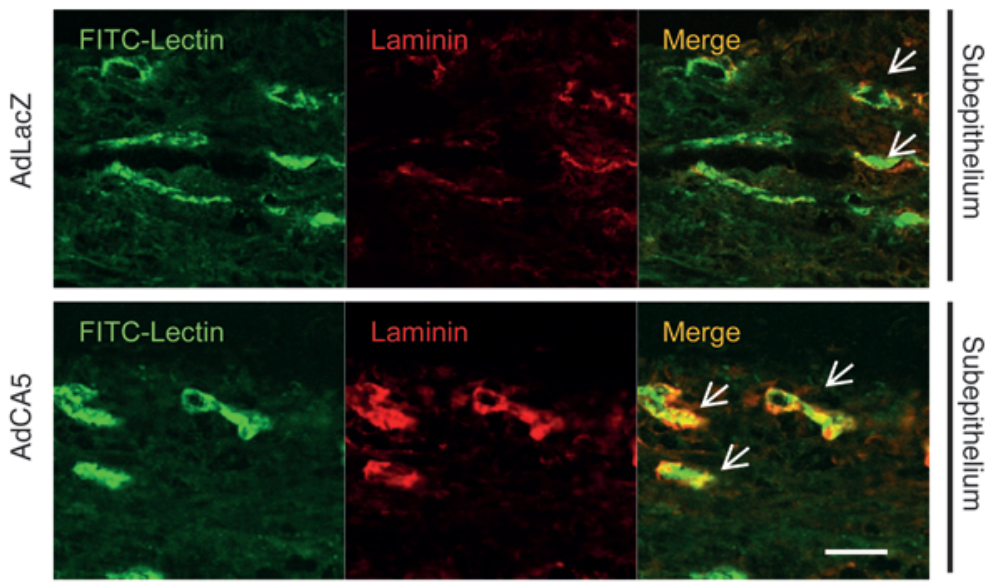

C

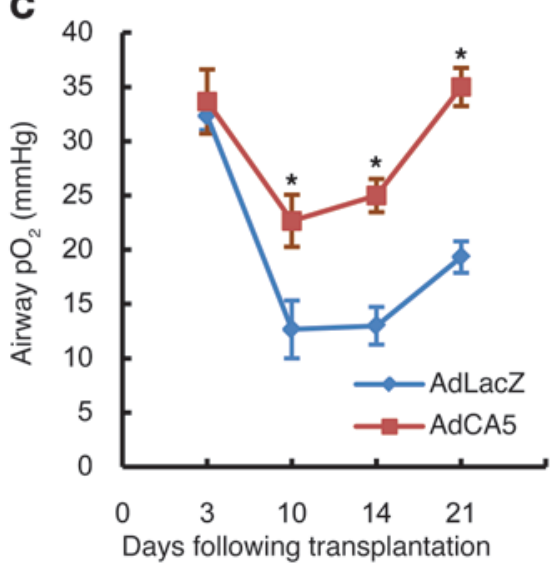

E
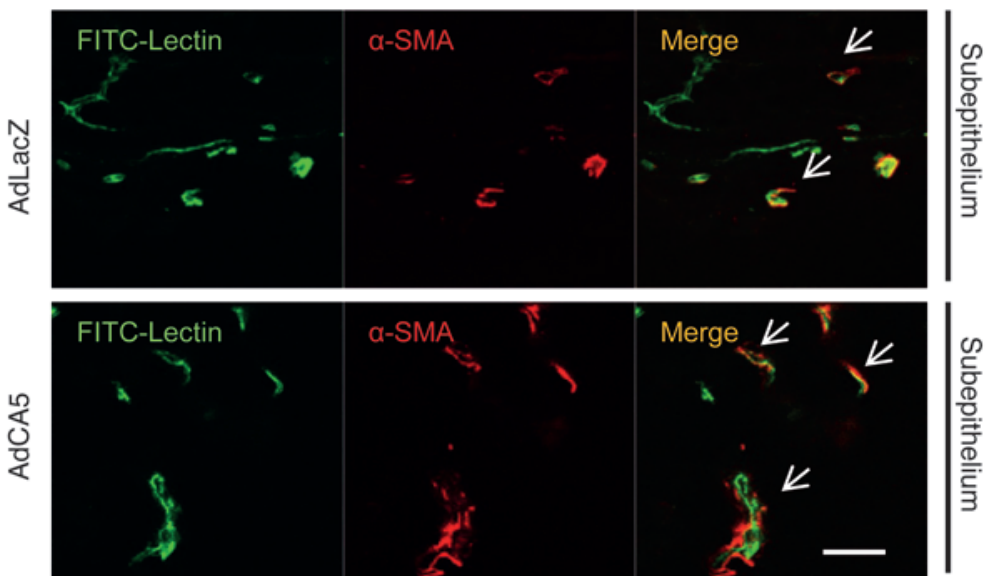

\section{Figure 4}

HIF-1 $\alpha$ gene transfer prolongs microvascular perfusion of airway allograft and alleviates tissue hypoxia. (A) Vascular perfusion of AdLacZ control adenovirus vector-treated allografts is lost at day 10 , day 12 , and day 14 , followed by partial revascularization at day 21 . However, perfusion of AdCA5-treated allografts is maintained at both day 10 and day 12, followed by partial loss at day 14 and near complete revascularization at day 21 .

(B) Percentage of perfusion area of AdLacZ- or AdCA5-treated allografts was calculated. $\left(n=4-6\right.$; $\left.{ }^{*} P<0.05\right)$. (C) AdCA5-treated allografts have higher tissue $\mathrm{pO}_{2}$ levels compared with AdLacZ-treated grafts. ( $n=4-6$; ${ }^{*} P<0.05$ at individual time points). (D) FITC-conjugated lectin perfusion and laminin staining show that AdCA5-treated chronically rejected day-21 allografts (lower panel) have better laminin-invested vessels (arrows) than AdLacZ-treated grafts (top panel). (E) FITC-conjugated lectin perfusion and $\alpha$-SMA staining show that AdCA5-treated chronically rejected day-21 allografts (lower panel) have more pericyte-covered vessels (arrows) than AdLacZ-treated grafts (top panel). Scale bars: $100 \mu \mathrm{m}(\mathbf{A}) ; 20 \mu \mathrm{m}$ (D and E). Data are shown as mean \pm SEM. 
Utilizing actin-GFP mice as recipients, there was a significant infiltration of $\mathrm{GFP}^{+}$cells in the acutely rejecting airway allograft, but only a small percentage $(15.0 \% \pm 3.3 \%)$ of $\mathrm{Hifl}^{+}$cells were of recipient origin (Figure 3C). These findings cumulatively demonstrate that increased Hifla expression in rejecting airway allografts is primarily attributable to donor endothelial cells.

To prove that HIF- $1 \alpha$ is required for the repair of damaged allograft microvasculature during acute rejection, we established an Hifla conditional knockout (CKO) model (Supplemental Figure 5). Hif1a CKO mice (on a B6 genetic background) were phenotypically normal, and Hifla CKO airways appeared histologically normal at baseline (data not shown). Transplantation of control B6 or Hif1a CKO tracheas into Balb/c mice showed that in both cases, the trachea graft was perfused by day 4 (Figure 3D), suggesting that the formation of vascular anastomosis between the donor and the recipient is independent of donor Hifla expression. In contrast to control WT allografts, which lost perfusion at day 10, Hif1a CKO tracheas were ischemic by day 8 ( $95 \%$ perfusion in control vs. $\sim 20 \%$ perfusion in Hifla CKO trachea). Moreover, revascularization of Hifla CKO trachea was significantly impaired in chronic rejection ( $\sim 50 \%$ perfusion in control vs. $\sim 5 \%$ in Hifla CKO trachea) (Figure 3, D and E). These data suggested that HIF- $1 \alpha$ is required not only for maintaining the integrity of the injured microvasculature but also for revascularization of the airway following alloimmune rejection. To identify putative molecular mechanisms implicated in the deterioration of microvascular health in acutely rejected Hifla CKO allografts, we examined expression levels of angiogenic growth factors and found that Sdf1 and Plgf (particularly Sdf1) were significantly decreased (Figure 3F). Administration of AMD3100, a CXCR4-specific antagonist capable of interrupting the SDF1/CXCR4 signaling pathway, also led to the loss of microvascular perfusion at day 8 (i.e., 2 days earlier than control loss of perfusion; Supplemental Figure 6). These data together suggest that the HIF-1 $\alpha /$ SDF1/CXCR4-mediated signaling pathway may play an essential role in the repair of the microvasculature of acutely rejected allografts.

It has been shown that the epithelium of a tissue-engineered trachea tends to be normal when there is a better subepithelial microvascular supply (32); we have demonstrated that loss of functional microvasculature at day 10 is correlated with denudation of the epithelium (8). Thus, we sought to determine whether accelerated loss of microvascular perfusion leads to earlier epithelial denudation. Examination of day-8 WT and Hifla CKO allografts was carried out by E-cadherin (a specific marker for epithelial cells) staining following FITC-lectin perfusion. The control day- 8 allografts have intact elongated epithelium with normal subepithelial microvascular perfusion (Figure $3 \mathrm{G}$ ). In contrast, the epithelium of the day-8 Hifla CKO trachea was flat, disorganized, and barely sufficient to cover the basement membrane (Figure 3G). Recruitment of Tie $2^{+}$cells to Hifla CKO donors was significantly diminished, with only half the recipient Tie $2^{+}$cells of that exhibited by controls (Figure 3, H and I), suggesting that Hifla-mediated signaling is required for recruitment of Tie $2^{+}$angiogenic cells.

Transient Hifla gene overexpression prolongs microvascular perfusion of airway allograft and alleviates tissue hypoxia. Given that Hif1 a deficiency led to an accelerated loss of airway microvasculature, HIF- $1 \alpha$ overexpression in the donor was next evaluated for its possible vasoprotective effects. Administration of AdCA5, an adenovirus encoding a constitutively active form of HIF-1 $\alpha$, was previously demonstrated to improve recovery of limb perfusion following unilateral femoral artery ligation in both diabetic and nondiabetic mice $(12,14)$. To determine the effect of AdCA5 on alloimmuneinjured microvasculature, time course of LacZ expression in AdLacZ-treated trachea was carried out by X-gal staining to estimate the transduction efficiency of the adenovirus vector. At day 4 following transplantation, both the epithelial layer and the adventitia were strongly stained blue, whereas fewer $\mathrm{LacZ}^{+}$cells were found in the subepithelial area (Supplemental Figure 7A). LacZ ${ }^{+}$ cells become less abundant at day 8 (Supplemental Figure 7B) and nearly undetectable at day 12 (Supplemental Figure 7C), suggesting that the expression of exogenous HIF-1 $\alpha$ in this model is short-lived. Microvascular perfusion of AdCA5-treated tracheal allograft was prolonged by more than 48 hours to day 12 ( $95 \%$ perfusion), followed by partial loss at day $14(\sim 40 \%$ perfusion) and near complete revascularization at day $21(\sim 90 \%$ perfusion) (Figure 4A). However, control adenovirus AdLacZtreated allografts lost perfusion from day 10 through day 14 ( $<15 \%$ perfusion), followed by partial perfusion recovery $(\sim 40 \%$ perfusion) at day 21 (Figure 4, A and B).

Airway $\mathrm{PO}_{2}$ has been successfully employed as a parameter to estimate microvascular perfusion status (8). Consistent with the perfusion improvement by AdCA5 treatment, airway tissue $\mathrm{pO}_{2}$ levels (as assessed by a fiber optic probe placed against the airway epithelial luminal surfaces) were augmented from day 10 through day 21 following transplantation (Figure 4C). Improved tissue oxygenation may be achieved by increased density and maturation of vessels (33). We therefore sought to determine whether HIF-1 $\alpha$ overexpression promoted microvascular normalization. FITClectin perfusion coupled with laminin (a basement membrane protein) and $\alpha$-SMA (to identify pericytes) staining showed that AdCA5-treated donor microvasculature had better laminin coverage $(56.5 \% \pm 4.8 \%$ in AdLacZ-treated allografts vs. $85.6 \% \pm 4.4 \%$ in AdCA5-treated grafts; $n=6 ; P<0.05$; Figure 4D) and more pericyte-covered vessels $(31.2 \% \pm 5.0 \%$ in AdLacZ-treated allografts vs. $62.1 \% \pm 6.5 \%$ in AdCA5-treated grafts; $n=5 ; P<0.05$; Figure 4E), signifying that AdCA5-treated airway microvasculature has a more mature and relatively stable phenotype.

Transient HIF-1 $\alpha$ gene overexpression accelerates replacement of donor endothelial cells by recipient-derived cells. $\mathrm{CD}^{+}$and $\mathrm{CD}^{+}$lymphocytes are the main cells that are involved in alloimmune-mediated endothelial cell injury (34). To exclude the possibility that improvement of microvascular perfusion by AdCA5 treatment is because of its effect on preventing infiltration of $\mathrm{CD}^{+}$and $\mathrm{CD}^{+}$ lymphocytes, we gated on $\mathrm{CD}^{+}$mononuclear cells and assessed $\mathrm{CD}^{+}$and $\mathrm{CD}^{+}$populations in the day- 6 rejecting allograft. Flow cytometry analysis showed that the percentage of both $\mathrm{CD}^{+}$and $\mathrm{CD}^{+}$lymphocytes recruited to the allografts was comparable after AdLacZ (Figure 5A) versus AdCA5 (Figure 5B) treatment. The majority of infiltrating $\mathrm{CD}^{+}$cells in both groups were CCR7-, which is consistent with these infiltrating $\mathrm{T}$ cells being effector memory $\mathrm{T}$ cells (data not shown). The degree of immune response can often be estimated by the local expression levels of proinflammatory cytokines and chemokines. Although increased expression of MCP-1, IL-1 $\beta$, and IL- 6 has been shown to be positively associated with the development of BOS and lung fibrosis (35-37), AdCA5 treatment did not significantly affect the level of these genes (Figure 5C). Augmented production of angiogenic growth factors is the main mechanism by which hypoxia induces neovascularization (9). Examination of day-6 allografts showed that AdCA5treated tracheas expressed significantly higher levels of Sdf1, Plgf, 
A

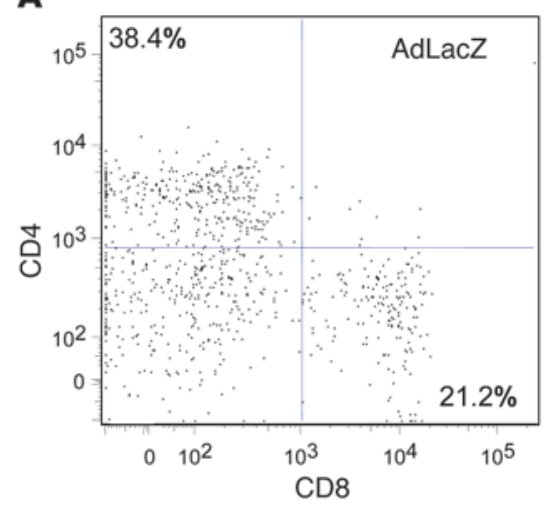

B

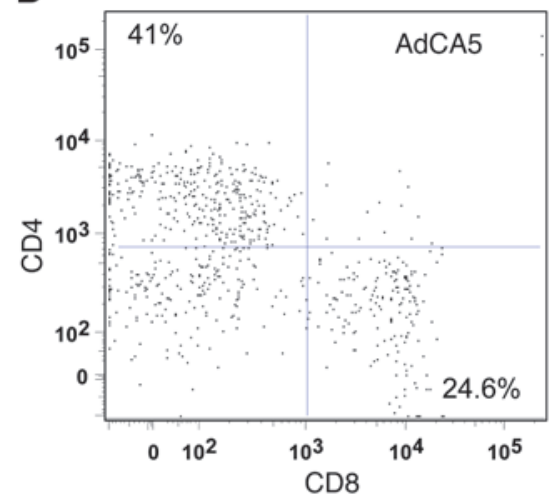

C

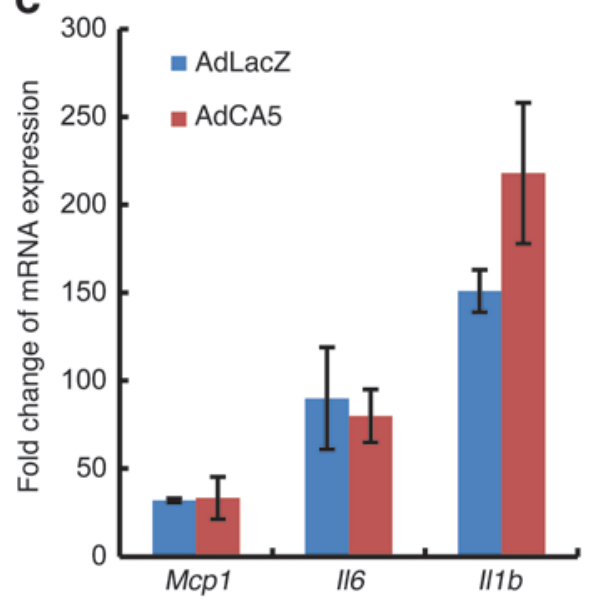

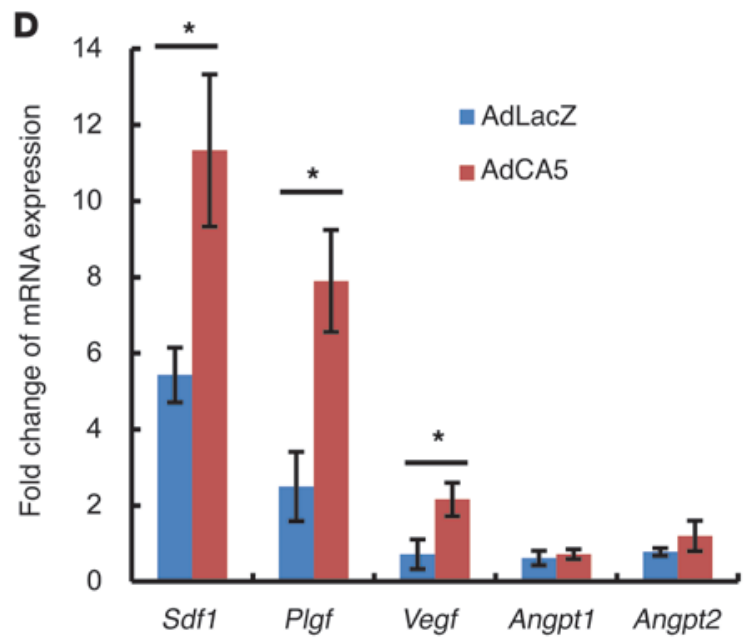

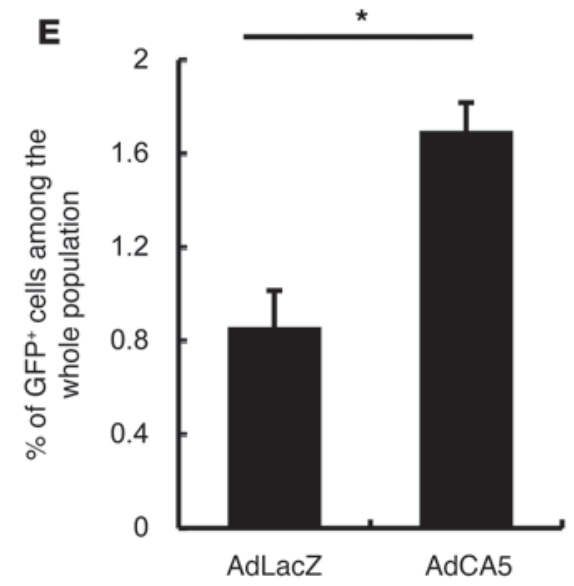

$\mathbf{F}$

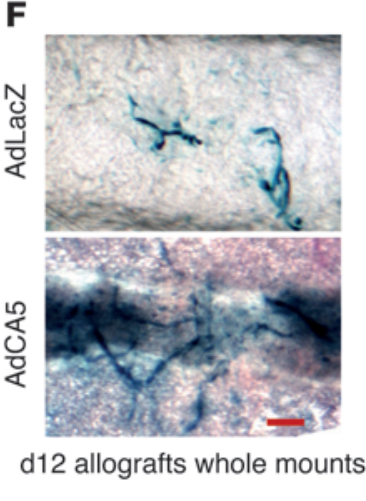

AdLacZ

AdCA5

Figure 5

HIF-1 $\alpha$ gene transfer increases expression of angiogenic factors and accelerates endothelial cell replacement by recipient-derived cells. (A and B) Flow cytometry analysis of day- 6 allografts shows that infiltrated CD4+ and CD8+ T lymphocytes are comparable in AdLacZ-treated (A) or AdCA5-treated (B) samples. The percentages indicate CD4+ or CD8 ${ }^{+}$cells among $C D 3^{+}$mononuclear cells. (C and D) Real-time RT-PCR analysis of day- 6 allografts demonstrated that the expression of proinflammatory cytokines is not significantly different between AdLacZ and AdCA5 treatment $(\mathbf{C})$, but expression of proangiogenic factors Plgf, Sdf1, and Vegf is significantly different (D) $\left(n=6\right.$; ${ }^{*} P<0.05$ of each proangiogenic factor). (E) AdLacZ- or AdCA5-treated Balb/c tracheas were transplanted into FVB (Tie2-EGFP) mice, and day- 6 allografts were analyzed by flow cytometry. AdCA5-treated allografts recruit higher levels of GFP+ cells in comparison with AdLacZ-treated grafts $\left(n=6,{ }^{*} P<0.05\right)$. (F) AdLacZ- or AdCA5-treated Balb/C tracheas were transplanted into FVB (Tie2-LacZ), mice and day-12 allografts were analyzed by X-gal staining. AdCA5-treated allografts have more recipient-derived Tie2 ${ }^{+}$vasculature (blue). Scale bar: $20 \mu \mathrm{m}$. Data are shown as mean \pm SEM.

and Vegf, but the levels of angiopoietin 1 and angiopoietin 2 were not elevated (Figure 5D). These data together suggested that the effect of AdCA5 on donor microvasculature was not likely achieved simply by attenuating the alloimmune response.

The increased expression of ischemia-induced angiogenic factors in HIF- $1 \alpha$-transduced OTT provided a molecular basis for the recruitment of circulating angiogenic cells. It was therefore of interest to determine whether increased expression of HIF- $1 \alpha$ promoted recruitment of recipient-derived Tie $2^{+}$cells to the airway allograft. Tracheas from $\mathrm{Balb} / \mathrm{c}$ mice were transplanted into mice with the transgene Tie2-GFP. Recruitment of recipient $\mathrm{GFP}^{+}$cells to the AdCA5-treated donor was significantly increased in acutely rejecting OTTs (Figure 5E). To determine whether increased expression of HIF- $1 \alpha$ could accelerate replacement of donor endothelial cells by recipient-derived cells, Tie2-LacZ mice were used as recipients. On day 12 of acute rejection, a period when donor-derived vessels are significantly injured, significantly more $\beta$-gal ${ }^{+}$(recipientderived) vasculature was found in the intercartilaginous part of the trachea in AdCA5-treated samples compared with AdLacZ controls (Figure 5F). These data suggest that overexpression of HIF-1 $\alpha$ in airway allografts during the acute phase of rejection may promote graft vascular repair by accelerating the microvascular investment by recipient-derived cells. This finding also suggested that in the day-14 AdCA5-treated acutely rejecting allografts, the perfused vessels in the cartilaginous part were mainly of recipient origin, possibly because only those cells were likely to survive the unmitigated acute alloimmune response (Figure 4A).

Transient overexpression of HIF-1 $\alpha$ prolongs the time window for the acutely rejecting tracheal allograft to be rescued from chronic rejection. We previously demonstrated that the loss of a functional microvasculature identifies allografts that can no longer be rescued by immunotherapy; in this prior study, failing microvessels were clearly documented by 
A

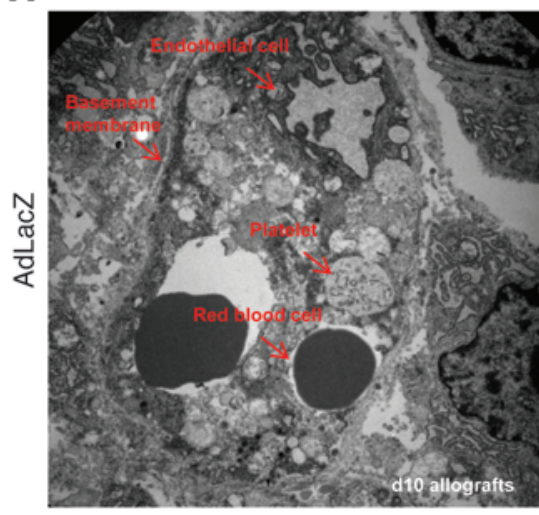

C
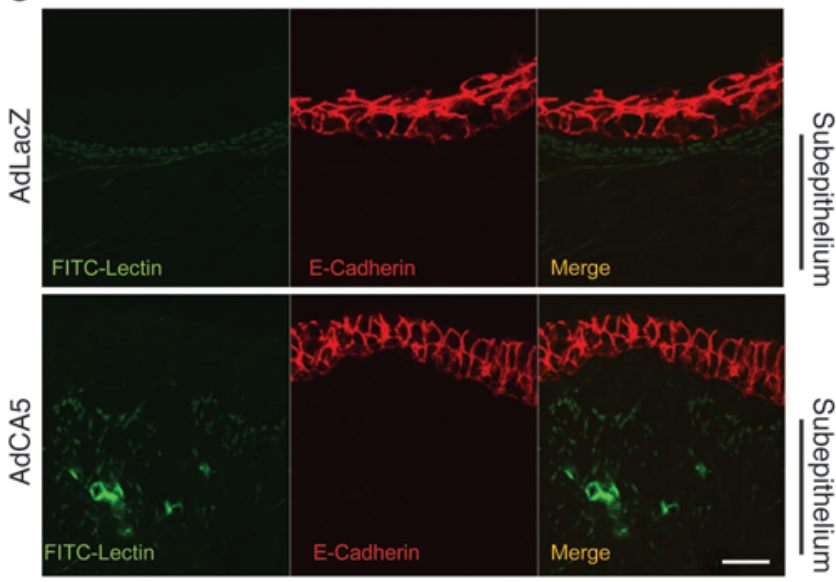

d12 allografts
B

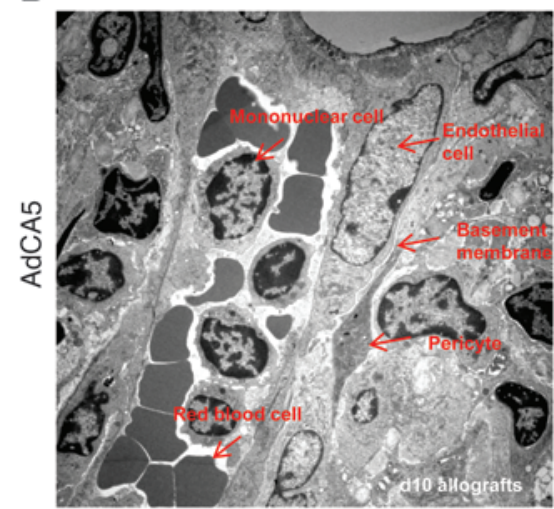

D

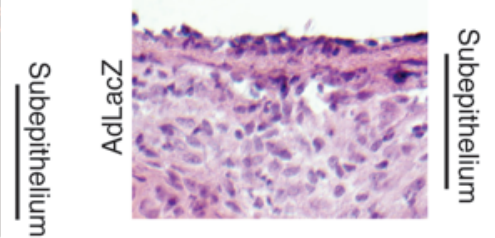

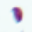

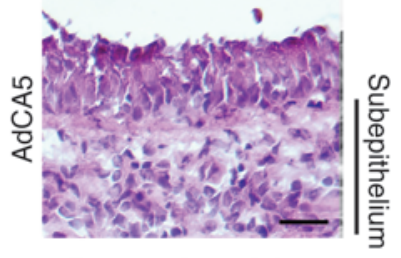

Retransplanted d12 allografts (recipients treated with anti-LFA-1/ anti-CD40L for 28 days)

\section{Figure 6}

HIF-1 $\alpha$ gene overexpression extends the time window for acutely rejecting allografts to be rescued from chronic rejection. (A) Electron micrograph demonstrating abnormal appearance of endothelial cells with disrupted basement membrane and thrombus within the damaged vessel in day-10 allografts treated with AdLacZ. Original magnification, $\times 4,500$. (B) Electron micrograph demonstrating normal appearance of vascular endothelial cells with relatively intact basement membrane and the existence of pericytes around the endothelial cells in day-10 allografts treated with AdCA5. (C) FITC-conjugated lectin perfusion coupled with IF staining of E-cadherin shows a flattened epithelial layer (red) and absence of subepithelial microvascular perfusion (green) in AdLacZ-treated samples. AdCA5-treated samples show a columnar epithelium with subepithelial vessel perfusion. (D) Day-12 allografts treated with AdLacZ or AdCA5 were retransplanted into naive B6 mice treated with immunosuppression, and the allografts were harvested 28 days following retransplantation. H\&E staining results reveal that AdLacZ-treated retransplants developed chronic rejection (top panel), and acute rejection was successfully reversed in AdCA5-treated retransplants (lower panel). Scale bars: $20 \mu \mathrm{m}$.

electron microscopy (8). To further determine whether the benefit of AdCA5 treatment could be demonstrated at the ultrastructural level, transmission electron microscopy showed that AdCA5-treated day10 allografts appeared significantly healthier compared with AdLacZtreated samples, with normally appearing endothelial cells, relatively intact basement membranes, and complete pericyte coverage (Figure 6, $A$ and $B$ ). These findings suggested that the microvasculature of AdCA5-treated allografts was still functional and less leaky after 10 days of rejection. Because AdCA5-treated day-12 allografts still had a fully perfused microvasculature, we reasoned that these allografts might still be able to be rescued with immunosuppression. Examination of day-12 allograft histology showed that in control AdLacZ-treated trachea, subepithelial microvascular perfusion is lost, and the epithelium is flat. In contrast, AdCA5-treated allografts had a relatively normal columnar epithelium with perfused subepithelial microvasculature (Figure 6C). These data again demonstrated the strong positive correlation between a functional microvasculature and a pseudostratified columnar phenotype in the overlying epithelium. AdLacZ- or AdCA5-treated acutely rejecting day-12 allografts were retransplanted into naive $\mathrm{B} 6$ mice treated with combined anti-CD40L and anti-LFA-1 immunosuppression as described (8). Twenty-eight days following retransplantation, only AdCA5-treated transplants demonstrated reversal of acute rejection to normal airway architecture; AdLacZ-treated airways, in contrast, developed subepithelial fibrosis with an abnormal layer of flat epithelium indicative of chronic rejection (Figure 6D). These data together demonstrate that HIF-1 $\alpha$ overexpression is able to synergize with immunosuppression to delay the onset of fibrosis and preserve airway architecture. 
A

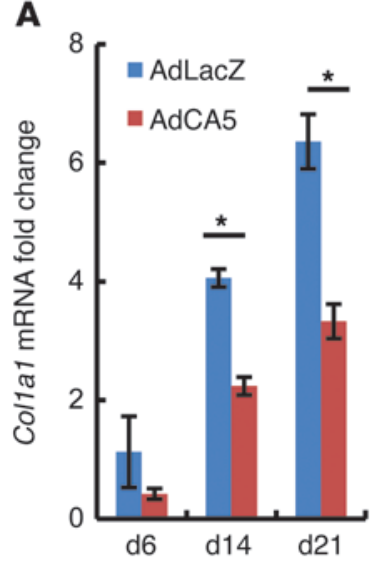

B

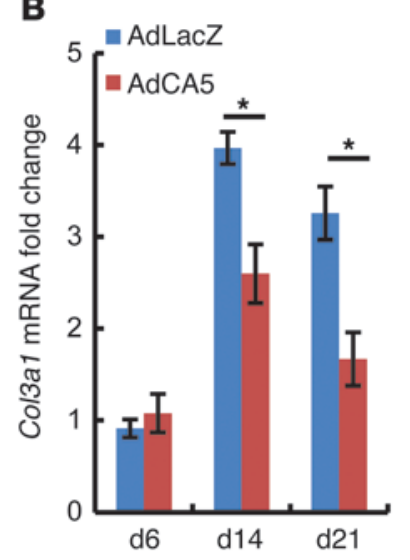

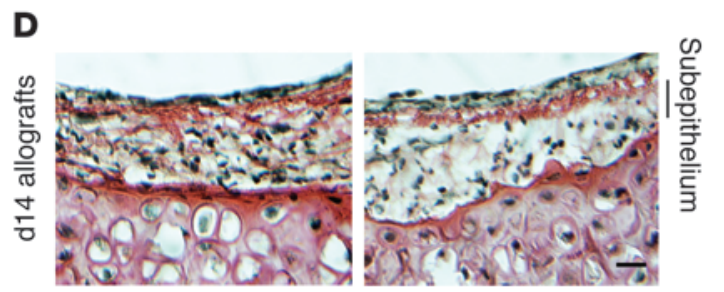

E

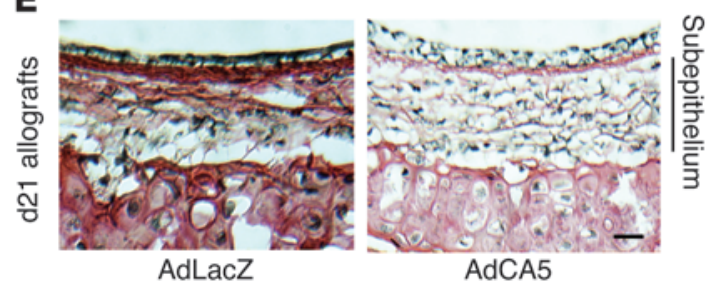

HIF-1 $\alpha$ overexpression attenuates fibrosis of airway allograft. To more fully elucidate the role of Hifla in the development of fibroproliferative disorders of the airway allograft, we assessed the degree of fibrosis of allografts treated with AdCA5 or AdLacZ. Collagen deposition is the key marker of lung fibrosis (38), and collagen I and III are the 2 main collagen types that deposit in a fibrotic lung (39). We therefore examined the gene expression levels of Col1a1 and Col3a1. Our data revealed that transcription of both Col1a1 and Col3a1 mRNA was significantly upregulated in control AdLacZ-treated allografts 14 and 21 days following transplantation. In contrast, we observed an approximately $50 \%$ decrease of both genes in AdCA5-treated allografts (Figure 7, $A$ and B). Collagen $V$ is a type of fibrillar collagen that has been implicated in the development of $\mathrm{OB}(40)$. Consistent with this clinical finding, examination of Colsa1 in the current model showed the diminished expression in protected AdCA5-treated allografts (Figure 7C). To further confirm that collagen gene expression was translated into protein, we next compared collagen protein deposition in tracheal allograft by picrosirius red staining. We observed that both at day 14 and day 21, AdCA5-treated allograft deposited significantly less collagen in the subepithelial area (Figure 7, D and E). These data together suggest that HIF-1 $\alpha$ overexpression is able to attenuate airway fibrosis following acute alloimmune injury.

\section{Discussion}

We found that the microvasculature of an airway subjected to prolonged alloimmune rejection had unusual structural features: the remodeled vessels do not have larger caliber arterioles or venules but

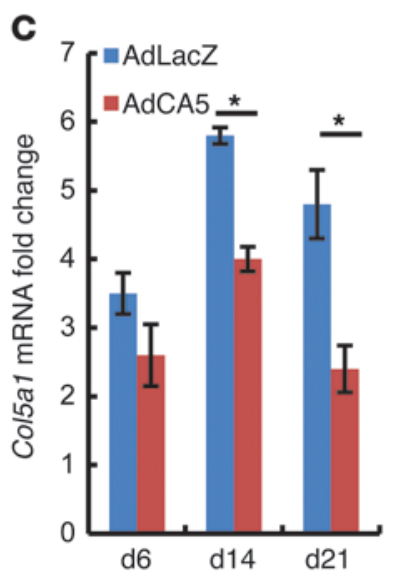

Figure 7

HIF-1 $\alpha$ gene overexpression attenuates fibrosis of airway allografts. (A-C) RT-PCR analysis of Col1a1, Col3a1, and Col5a1 mRNA expression shows AdCA5-treated allografts express diminished levels of Col1a1 (A), Col3a1 (B), and Col5a1 (C) at day 14 and day $21(n=6$; ${ }^{\star} P<0.05$ ). (D and E) Picrosirius red staining was used to estimate the levels of collagen protein in trachea allografts. Diminished collagen deposition is seen in AdCA5-treated day-14 (D, right panel) and day-21 (E, right panel) allografts. Scale bars: $20 \mu \mathrm{m}$. Data are shown as mean \pm SEM. instead are invested with tortuous vessels with relatively poor pericyte coverage that resemble unstable tumor vessels. These remodeled microvessels may be less efficient in the delivery of nutrients and oxygen to injured airways and may put the airway at increased risk for further fibrotic remodeling (41-43). Our data also suggest that the density of functional airway microvasculature may vary in different stages following transplantation. This finding is consistent with the Papworth Hospital autopsy study, which demonstrated a higher density of small caliber vessels with advancing BOS (4).

We found that recipient-derived $\mathrm{Tie}^{+}$cells are the principal endothelial population that replaces damaged donor endothelium. Interestingly, recipient $\mathrm{Tie}^{+}$cells recruited to the allograft early after transplantation comprise not only $\mathrm{Tie}^{+} \mathrm{CD} 45^{-} \mathrm{CD} 31^{+}$ progenitor cells with endothelial origin but also $\mathrm{Tie}^{+} \mathrm{CD} 45^{+} \mathrm{CD} 31^{-}$ TEMs and a population of Tie2 ${ }^{+} \mathrm{CD} 45^{-} \mathrm{CD} 31^{-}$cells. Moreover, expression levels of HIF-1 $\alpha$ positively correlate with the number of migrating Tie $2^{+}$cells in the allografts undergoing acute rejection, suggesting that the recruitment and retention of $\mathrm{Tie}^{+}$cells are mediated by HIF- $1 \alpha$-induced expression of proangiogenic factors such as PLGF, VEGF, and SDF1 (13, 44-47) (modeled in Figure 8A). TEMs exhibit paracrine proangiogenic activities $(16,18)$, suggesting that in an airway subjected to acute rejection, $\mathrm{Tie}^{+}$cells participate in microvascular repair beyond physically integrating into the donor endothelium. In a mouse mammary tumor model, TEMs account for most of the proangiogenic activity of myeloid cells and play an essential role in tumor neovascularization (16). In the chronically rejected allograft, less than $2 \%$ 
A

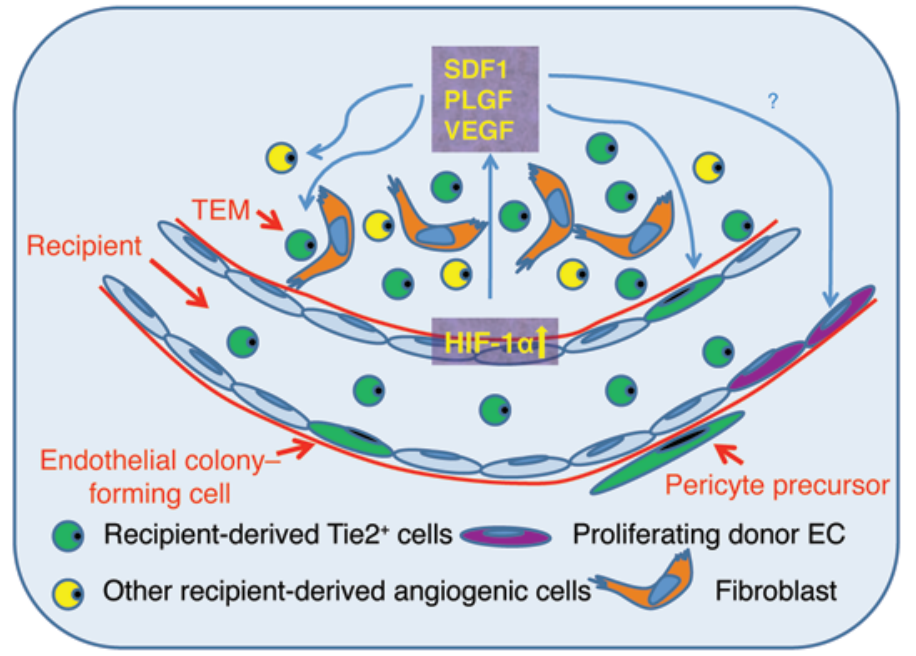

B

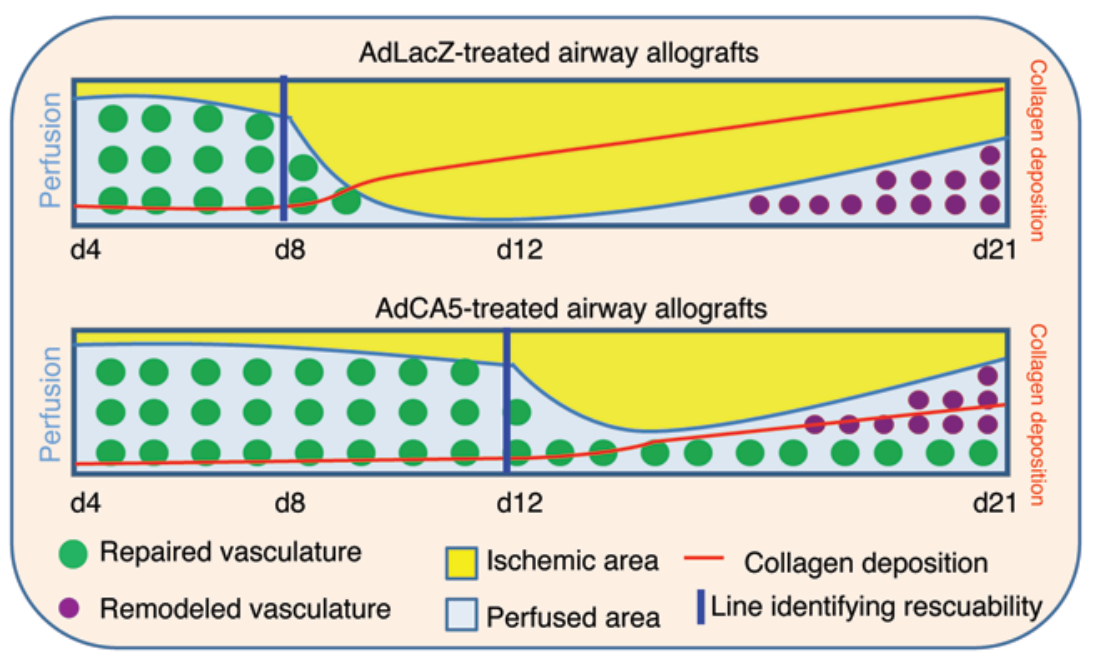

\section{Figure 8}

Model of how HIF-1 $\alpha$ promotes airway microvascular repair and prevents fibrosis. (A) Scheme of recipient-derived Tie2 ${ }^{+}$cells contributing to microvascular repair in allograft rejection. Increased allograft hypoxia during rejection leads to increased expression of HIF- $1 \alpha$ in endothelial cells, which further induces expression of proangiogenic factors that promote the recruitment and retention of recipient-derived angiogenic cells, including Tie2 ${ }^{+}$cells (green) and other non-Tie2+ cells (yellow). Enhanced expression of HIF- $1 \alpha$ accelerates vascular repair. (B) Scheme of the effects of HIF-1 $\alpha$ gene overexpression on airway perfusion and fibrosis. Perfusion is reestablished at day 4 following transplantation in both AdLacZ- and AdCA5treated OTT after microvascular reconnection between the donor and the recipient. Progressive microvascular damage leads to a complete loss of perfusion at around day 10, followed by a partial microvascular reestablishment at day 21 in AdLacZ-treated allografts. Reestablished microvessels are phenotypically distinct from normal ones. In contrast, microvascular perfusion is prolonged to day 12 in AdCA5treated allografts. This anatomic finding identifies day-12 allografts as "rescuable" to normal architecture by immunosuppression (versus day 8 for control airways; blue line). The onset and the intensity of the collagen deposition are significantly delayed and diminished respectively in AdCA5-treated airways (red line). Alleviation of fibrosis in HIF- $1 \alpha$-overexpressed airways is likely due to the decreased burden of tissue hypoxia (ischemia $\times$ time duration), which is represented by the yellow area. of Tie $2^{+}$cells were TEMs (data not shown), suggesting that TEMs are preferentially recruited to the donor during acute rejection where they may participate in microvascular repair and, following this, TEMs are either transformed into other cells types, migrate out of the graft, or die. The data indicate that TEMs play a minimal role in vessel remodeling during chronic rejection. Lineage tracing of Tie $2^{+}$cells showed that recipient-derived Tie $2^{+}$cells (most likely from the Tie ${ }^{+} \mathrm{CD} 45^{-} \mathrm{CD} 31^{-}$population) also differentiate into pericytes, which may help to stabilize newly formed vessels. The heterogeneous $\mathrm{Tie}^{+}$cells play various roles in vascular repair, which include functioning as TEMs, endothelial cells, and pericytes; these cells work in a cooperative fashion to promote microvascular recovery (Figure 8A). Other non-Tie2 ${ }^{+}$proangiogenic cells with hematopoietic origin have been previously shown to contribute to neovascularization in various models $(46,48,49)$ and it is possible that some of those cells may also participate in the repair of alloimmune-injured microvasculature (Figure 8A). Activation and proliferation of local endothelial cells by proangiogenic growth factors are essential for neovascularization $(9,50)$. In an allograft undergoing acute rejection, however, this may be less important compared with the replacement of donor endothelial cells by recipient-derived circulating cells.
We designed a proof-of-principle experiment to show that promoting microvascular repair during phases of acute rejection is able to delay the allograft's development of chronic rejection. Airway microvascular perfusion analysis showed that perfusion of both control and Hifla CKO allografts began on day 4, indicating that donor Hifla expression is not required to initiate angiogenesis that is involved in early airway microvascular anastomosis formation. Instead, other signaling pathways activated by the inflammatory microenvironment of the allograft might be sufficient to start the process of microvascular reconnection between the donor and the recipient. The early loss of perfusion at day 8 in Hif1a CKO allografts and the prolonged perfusion of HIF-1 $\alpha-$ overexpressing allografts compared with control allografts suggest that HIF-1 $\alpha$ accumulation as a physiological response to ischemic stress is essential for maintaining a functional donor microvasculature. Furthermore, these experiments demonstrate that augmented expression of HIF- $1 \alpha$ by gene therapy is able to promote neovascularization and repair of the damaged donor microvasculature (Figure 8B).

Examination of AdCA5-treated acutely rejecting allografts revealed an accelerated donor endothelial cell replacement by the recipient-derived cells, suggesting that HIF-1 $\alpha$ gene trans- 
fer may promote endothelial cell accommodation and decrease donor immunogenicity (28). The accelerated loss of perfusion observed in animals undergoing CXCR4 blockade suggests that the HIF-1 $\alpha /$ SDF1/CXCR4 signaling pathway is important in maintaining airway perfusion during rejection. Another important phenotypic improvement of the donor microvasculature after HIF- $1 \alpha$ gene transfer is increased endothelial cell coverage by pericytes and basement membrane, 2 features that have been shown to be critical for increased vessel stability $(25,26)$. Therefore, HIF-1 $\alpha$ gene transfer can lead to a more mature and stable microvasculature to both prevent and alleviate allograft ischemia. Future studies can address whether increasing HIF-1 $\alpha$ in recipient tissues is also beneficial.

HIF-1 $\alpha$ gene transfer not only significantly diminished collagen deposition, but also prolonged the time window for the allograft to be rescued from chronic rejection with immunosuppression. Although AdCA5 therapy downregulated collagen isoforms, including the potentially key collagen $\mathrm{V}$ implicated in BOS (40), airway fibrosis was not completely prevented by HIF-1 $\alpha$ overexpression in the absence of immunosuppression (Figure $8 \mathrm{~B}$ ). This is probably due to the overwhelming alloimmune response. We observed that, even with HIF-1 $\alpha$ gene therapy, the airway allograft still undergoes a period of hypoxia, albeit with decreased degree and duration in comparison with control treatment (Figure 8B). This finding strongly suggests that the product of ischemic area multiplied by the time duration of an allograft may be used as a parameter to predict the development and the severity of fibrosis. Lung is unique among solid organ transplants in that it is not routinely reattached to the systemic circulation by bronchial arterial reconnection at the time of surgery. Blood supply to the airways in lung transplant recipients is therefore presumably supplied by the deoxygenated pulmonary artery circulation (51). Therefore, from the onset, lung transplant airways have an impaired microcirculation due to the lack of blood supply from the bronchial artery, which results in relative airway tissue hypoxia (23). We have hypothesized that baseline airway hypoxia may be a diathesis for chronic rejection in lung transplant recipients (23). The results of the current study provide further compelling preclinical evidence to support those clinical findings as well as the hypothesis that hypoxia and ischemia during acute rejection put the allograft at risk for chronic rejection.

Organ dysfunction caused by fibrosis following alloimmune injury is the principal limiting factor for long-term survival of transplanted solid organs. While suppression of alloimmune responses has been successfully used to prevent acute rejection, it has not been sufficient for indefinitely preventing the development of chronic rejection. Several lines of evidence now suggest that loss of the microvascular circulation may signal the development of chronic rejection. This current study provides, to our knowledge, the first demonstration that during acute rejection, promoting the microvascular repair of an allograft through gene therapy can delay and attenuate fibrosis. Therapies that promote graft microvascular integrity, administered with heightened immunosuppression during acute rejection episodes, could effectively limit chronic rejection.

\section{Methods}

Mice. All animal procedures were approved by Stanford's Administrative Panel on Laboratory Animal Care (APLAC) and/or the VA Palo Alto Institutional Animal Care and Utilization Committee (IACUC). All mice including C57BL/6J (B6; H-2 $)$, Balb/C (H-2 $)$, FVB/NJ (H-2q), FVB/N-Tg (TIE2-lacZ) 182Sato/J, FVB-Tg (TIE2GFP287Sato/J), B6.Cg-Tg (Tek-cre) 12Flv/J, B6.129X1-Gt(ROSA)26Sortm1(EYFP)Cos/J, B6.Cg-Tg(CAG-cre/ Esr1*)5Amc/J, and B6.129-Hif1atm3Rsjo/J were purchased from the Jackson Laboratory.

For Tie2 lineage-tracing studies, ROSA26EYFP reporter mice were crossed with mice expressing Tie2-Cre (Supplemental Figure 2A). To create Hifla CKO, mice with loxP sites on both sides of exon 2 of the Hifla gene (Hif1 loxp/loxP $)$ were crossed with mice expressing tamoxifen-inducible Cre recombinase under the control of the CAG promoter (Cag-Cre-ER) (Supplemental Figure 4A). Mice with the transgenes Cag-Cre-ER, Hif1 ${ }^{W T / W T}$, and Cag-Cre-ER, Hif1 $1^{\text {loxP/loxP }}$, were used as control and Hifla CKO respectively. Mice carrying Cag-Cre-ER and floxed Hifla genes were treated with tamoxifen for 5 consecutive days at a dose of $120 \mathrm{mg} / \mathrm{kg}$ followed by at least 2 more days to allow sufficient recombination prior to their use in studies. Tamoxifen, which activates the Cre recombinase, efficiently recombined the Hifla gene (Supplemental Figure 5B).

Tracheal transplantation. Balb/C, B6, and FVB mice were used as donors or recipients as described. Basic surgical procedures of tracheal transplantation were carried out as previously described (8). Briefly, both donor and recipient mice were anesthetized with $50 \mathrm{mg} / \mathrm{kg}$ ketamine and $10 \mathrm{mg} / \mathrm{kg}$ xylazine. 5- to 7-ring tracheal segments were removed from donor mice that were matched for recipient age and sex. The donor tracheas were stored in PBS on ice before transplantation. For adenovirus gene-transfer experiments, donor tracheas were incubated in adenovirus solution $\left(2 \times 10^{12} \mathrm{vp} / \mathrm{ml}\right)$ for 60 minutes at $4^{\circ} \mathrm{C}$ before transplantation. A short incision was made in the midline of the neck region of the recipient. The strap muscles were then bluntly divided and pulled aside by 3-0 suture, which allowed clear exposure of the laryngotracheal complex. After the recipient trachea was transected, donor trachea was sewn in with 10-0 nylon sutures, and the skin was closed with 5-0 silk sutures.

Tissue preparation and immunohistochemistry. For whole-mount tracheal microvascular analysis, mice were injected with $100 \mu \mathrm{l}$ of FITC-conjugated tomato lectin (Vector Laboratories) at a concentration of $1 \mathrm{mg} / \mathrm{ml}$. After 5 minutes of circulation, mice were perfused with 1\% PFA diluted in PBS for about 2 minutes until the outflow of the solution turned clear. The tracheas were then harvested, put in $1 \%$ PFA for 1 hour at $4{ }^{\circ} \mathrm{C}$, and washed 3 times with PBS. Whole tracheas were mounted on glass slides in Vectashield H-1200 mounting medium (Vector Laboratories). Frozen sections were used for other immunohistochemistry analysis. Trachea samples were snap-frozen in OCT solution (Sakura Finetek) after harvest, and OCT-embedded samples were stored at $-80^{\circ} \mathrm{C}$. Tracheas with EGFP autofluorescence or FITC-lectin perfusion were fixed with $4 \%$ PFA for 1 hour on ice, followed by incubation in a $30 \%$ sucrose solution overnight at $4{ }^{\circ} \mathrm{C}$ before OCT embedding. All procedures were done in a way to minimize exposure to light. 8 - $\mu \mathrm{m}$ sections were used for H\&E or immunofluorescence staining. Anti-CD31 (1:200; BD Pharmingen) or anti-VEGFR2 (1:100; R\&D) antibody was used to stain endothelial cells; anti-E-cadherin antibody (1:200; eBioscience) was used to stain epithelial cells; anti-NG2 (1:100; Invitrogen), anti- $\alpha$-SMA (1:200; Sigma-Aldrich) or anti-PDGFR $\beta(1: 100 ; R \& D)$ antibody was used to stain pericytes; anti- $\beta$-gal (1:50; eBioscience) and anti-GFP (1:100; Abcam) antibodies were used to stain $\beta$-gal and YFP respectively; antiVEGF (1:100; R\&D), anti-SDF1 (1:100; R\&D), anti-PLGF (1:100; R\&D), and anti-laminin (1:100; LifeSpan Biosciences) antibodies were used to stain Vegf, $S d f 1, P l g F$, and laminin, respectively. Secondary antibodies were labeled with the fluorochromes Alexa Fluor 488 or Alexa Fluor 594 (1:200; Invitrogen). Nuclei were stained by DAPI (Vector Laboratories). Photomicrographs were taken with a Zeiss LSM 510 laser scanning confocal microscope with Zeiss LSM Image Browser software. In those 
experiments with relative percentage calculations, positively stained cells were counted in 8 optical fields per section, and the percentages were calculated based on at least 5 sections from different samples.

Western blotting. Liquid nitrogen-cooled tracheas were pulverized with a liquid nitrogen-cooled BioPulverizer. Powdered tracheas were handhomogenized in $70 \mu \mathrm{l}$ of RIPA buffer with both protease and phosphatase inhibitors (Thermo Fisher Scientific). Extracts were incubated on ice for 30 minutes, followed by centrifugation at $4^{\circ} \mathrm{C}$ for 15 minutes at $18,000 \mathrm{~g}$. Extracts containing $35 \mu \mathrm{g}$ protein were separated on SDS gels and transferred to nitrocellulose membranes. Actin was used as an internal control. Membranes were incubated with anti-HIF-1 $\alpha$ (Novus), anti-SDF-1 $(\mathrm{R} \& \mathrm{D})$, or anti-actin (Sigma-Aldrich) at $4{ }^{\circ} \mathrm{C}$ overnight. Blots were incubated with the appropriate secondary antibodies and signals were detected by PhosphorImager analysis using ECL Plus (Amersham).

Flow cytometry. Single-cell suspension of allografts were prepared by incubating tracheas in $0.15 \%$ collagenase A (Roche Applied Science) for 40 minutes at $37^{\circ} \mathrm{C}$. Cells were then stained with the following antibodies: PE- and APC-conjugated CD31 and CD45 (eBioscience), FITC-conjugated CD4, APC-conjugated CD8, and PE-conjugated CD3 (BD Pharmingen). Propidium iodide was used to exclude dead cells. Stained cells were measured on FACSCalibur (BD Biosciences - Immunocytometry Systems) and analyzed with FlowJo software (Tree Star Inc.). Experiments were repeated at least 3 times, with 1 representative result presented.

$X$-gal staining. The staining was carried out by using the X-gal staining kit (Invitrogen). Briefly, the tracheas were first fixed in 2\% PFA for 1 hour on ice, followed by rinsing and incubating in solution A and solution B at room temperature per manufacturer's protocol. After incubation in X-gal solution at $37^{\circ} \mathrm{C}$ overnight, the whole trachea was placed on glass slide with mounting medium and covered with cover slips. Light microscopy was used to examine the staining.

Quantitative real time RT-PCR. Tracheal samples were first incubated in RNAlater solution (Invitrogen) overnight at $4{ }^{\circ} \mathrm{C}$. Total RNA was then isolated using the QIAGEN Shredder and RNeasy Mini Kit (QIAGEN) following the manufacturer's protocol. Total RNA $(1 \mu \mathrm{g})$ was reverse transcribed with Moloney murine leukemia virus reverse transcriptase (Invitrogen) and $5 \mu \mathrm{M}$ random hexamer primers according to the manufacturer's instructions. $2 \mu \mathrm{l}$ of 1 to 10 diluted reverse transcription reactions was added to quantitative real time RT-PCR (qRT-PCR) with $5 \mu$ l of $2 \times$ SYBR Green Master Mix (Applied Biosystems) and $100 \mathrm{nM}$ forward and reverse primers specific for the genes of interest in a total volume of $10 \mu l$. Detection was carried out with the ABI Prism 7700 sequence detector (Applied Biosystems). SDS analysis software (Applied Biosystems) was used to analyze the data. $18 \mathrm{~S}$ mRNA expression was used to normalize gene expression for sample-to-sample variation in input and reverse transcription efficiency. The $2^{-\Delta \Delta C t}$ method was used to calculate fold changes. Primers used are shown in Supplemental Table 1.

AMD3100 administration. AMD3100 (Sigma-Aldrich) was dissolved in PBS, and a total of $2.5 \mathrm{mg}$ per mouse was continuously delivered with the aid of a subcutaneous mini-osmotic pump (Model 2002; Alzet), starting day 1 following transplantation. Control animals were similarly implanted with saline-releasing pumps. OTTs were perfused, as already described, by FITC-conjugated lectin (Vector Laboratories) and harvested 4 days or 8 days following transplantation.

Tissue oximetry. Tissue oxygen content was measured by fluorescence quenching technique using OxyLab $\mathrm{pO}_{2}$ monitor (Oxford Optronix Ltd.) as previously described (8). The fiber optic probe was placed against the epithelial luminal surface to take measurements.

EM studies. OTTs were excised and fixed in $1.25 \%$ glutaraldehyde in $0.1 \mathrm{M}$ cacodylate buffer for 24 hours at room temperature. Samples were washed with $0.1 \mathrm{M}$ cacodylate buffer, postfixed in $2 \% \mathrm{OsO}_{4}$ buffered with $0.1 \mathrm{M}$ cacodylate for 2 hours. The samples were then embedded in epoxy resin, sectioned at $1 / 20$ micron, and stained sequentially with uranyl acetate and lead hydroxide. Stained sections were examined with a Phillips electron microscope (Advanced Microscopy Techniques) operating at $75 \mathrm{KV}$.

Delayed immunotherapy experiments. To study whether the alloimmuneinjured airway can be rescued by immunosuppression, a retransplantation coupled with immunosuppression model was used as previously described (8). Briefly, day-12 allografts treated with AdCA5 or AdLacZ were retransplanted into naive mice with anti-LFA- 1 and anti-CD40L combined immunotherapy. Twenty-eight days following retransplantation, the allograft was harvested and prepared for histological analysis.

Picrosirius red staining. Frozen sections were used for staining. Sections were fixed in a $1: 1$ acetone/methanol solution for 10 minutes at $-20^{\circ} \mathrm{C}$, followed by equilibration in distilled water for 2 minutes. Sections were then incubated in celestine blue for 5 minutes, followed by a 1-minute rinse in distilled water, and a 5-minute stain in hematoxylin. Sections were then washed in distilled water for 5 minutes and incubated in $0.1 \%$ sirius red (Sigma-Aldrich) for 1 hour. The sections were then rinsed in distilled water for 1 minute, followed by dehydration in 100\% ethanol 3 times (1 minute each); they were then cleared in xylene twice (1 minute each). Light microscopy was used to take photographs of picrosirius-stained sections.

Statistics. Statistical analysis was performed using 1-way ANOVA with Tukey's post test or 2-tailed Student's $t$ test, with a significance level of $P<0.05$.

\section{Acknowledgments}

This work was supported by NIH grants HL082662 and HL095686 (to M.R. Nicolls) and HL055338 and GM078494 (to G.L. Semenza) and a Stanford Postdoctoral Dean's Fellowship (to X. Jiang). The authors wish to gratefully acknowledge Mark Krasnow for advice and Norbert Voelkel for critically reading this manuscript.

Received for publication December 21, 2010, and accepted in revised form March 30, 2011.

Address correspondence to: Mark R. Nicolls, VA Palo Health Care System, Medical Service 111P, 3801 Miranda Ave., Palo Alto, California 94304, USA. Phone: 650.493.5000, ext. 69289; Fax: 650.849.0553; E-mail: mnicolls@stanford.edu.
1. Grossman EJ, Shilling RA. Bronchiolitis obliterans in lung transplantation: the good, the bad, and the future. Transl Res. 2009;153(4):153-165.

2. Belperio JA, Weigt SS, Fishbein MC, Lynch JP 3rd. Chronic lung allograft rejection: mechanisms and therapy. Proc Am Thorac Soc. 2009;6(1):108-121.

3. Weigt SS, Wallace WD, Derhovanessian A, Saggar R, Lynch JP, Belperio JA. Chronic allograft rejection: epidemiology, diagnosis, pathogenesis, and treatment. Semin Respir Crit Care Med. 2010. 31(2):189-207.

4. Luckraz H, et al. Microvascular changes in small airways predispose to obliterative bronchiolitis after lung transplantation. J Heart Lung Transplant. 2004;23(5):527-531.

5. Luckraz H, Goddard M, McNeil K, Atkinson C, Sharples LD, Wallwork J. Is obliterative bronchiolitis in lung transplantation associated with microvascular damage to small airways? Ann Thorac Surg. 2006;82(4):1212-1218.

6. Bishop GA, Waugh JA, Landers DV, Krensky AM, Hall BM. Microvascular destruction in renal transplant rejection. Transplantation. 1989;48(3):408-414.

7. Matsumoto Y, McCaughan GW, Painter DM, Bishop GA. Evidence that portal tract microvascular destruction precedes bile duct loss in human liver allograft rejection. Transplantation. 1993;56(1):69-75.

8. Babu AN, et al. Microvascular destruction identifies murine allografts that cannot be rescued from airway fibrosis. J Clin Invest. 2007;117(12):3774-3785.

9. Semenza GL. Regulation of oxygen homeostasis by hypoxia-inducible factor 1. Physiology (Bethesda). 2009;24:97-106.

10. Rey S, Semenza GL. Hypoxia-inducible factor-1dependent mechanisms of vascularization and vascular remodelling. Cardiovasc Res. 2010;86(2):236-242.

11. Wang GL, Jiang BH, Rue EA, Semenza GL. Hypoxia-inducible factor 1 is a basic-helix-loop-helix-PAS heterodimer regulated by cellular $\mathrm{O} 2$ tension. Proc 
Natl Acad Sci U S A. 1995;92(12):5510-5514.

12. Bosch-Marce M, et al. Effects of aging and hypoxiainducible factor- 1 activity on angiogenic cell mobilization and recovery of perfusion after limb ischemia Circ Res. 2007;101(12):1310-1318.

13. Rey S, et al. Synergistic effect of HIF-1alpha gene therapy and HIF-1-activated bone marrow-derived angiogenic cells in a mouse model of limb ischemia. Proc Natl Acad Sci U S A. 2009; 106(48):20399-20404.

14. Sarkar K, Fox-Talbot K, Steenbergen C, Bosch-Marce M, Semenza GL. Adenoviral transfer of HIF-1alpha enhances vascular responses to critical limb ischemia in diabetic mice. Proc Natl Acad Sci US A. 2009; 106(44):18769-18774.

15. Ceradini DJ, et al. Progenitor cell trafficking is regulated by hypoxic gradients through HIF-1 induction of SDF-1. Nat Med. 2004;10(8):858-864.

16. De Palma M, et al. Tie2 identifies a hematopoietic lineage of proangiogenic monocytes required for tumor vessel formation and a mesenchymal population of pericyte progenitors. Cancer Cell. 2005;8(3):211-226.

17. Lewis CE, De Palma M, Naldini L. Tie2-expressing monocytes and tumor angiogenesis: regulation by hypoxia and angiopoietin-2. Cancer Res. 2007;67(18):8429-8432.

18. Pucci $F$, et al. A distinguishing gene signature shared by tumor-infiltrating Tie2-expressing monocytes, blood "resident" monocytes, and embryonic macrophages suggests common functions and developmental relationships. Blood. 2009;114(4):901-914

19. Tepper OM, et al. Adult vasculogenesis occurs through in situ recruitment, proliferation, and tubulization of circulating bone marrow-derived cells. Blood. 2005;105(3):1068-1077.

20. Jackson KA, et al. Regeneration of ischemic cardiac muscle and vascular endothelium by adult stem cells. J Clin Invest. 2001;107(11):1395-1402.

21 . Tongers J, et al. Haeme oxygenase promotes progenitor cell mobilization, neovascularization, and functional recovery after critical hindlimb ischaemia in mice. Cardiovasc Res. 2008; 78(2):294-300.

22. Sato M, Keshavjee S, Liu M. Translational research: animal models of obliterative bronchiolitis after lung transplantation. Am J Transplant. 2009; 9(9):1981-1987.

23. Dhillon GS, et al. Lung transplant airway hypoxia: a diathesis to fibrosis? Am J Respir Crit Care Med. 2010;182(2):230-236.

24. Murakawa T, et al. Simultaneous LFA-1 and CD40 ligand antagonism prevents airway remodeling in orthotopic airway transplantation: implications for the role of respiratory epithelium as a modulator of fibrosis. J Immunol. 2005;174(7):3869-3879.

25. Jain RK. Normalization of tumor vasculature: an emerging concept in antiangiogenic therapy. Science. 2005;307(5706):58-62.

26. Gerhardt H, Semb H. Pericytes: gatekeepers in tumour cell metastasis? J Mol Med. 2008;86(2):135-144.

27. Lagaaij EL, Cramer-Knijnenburg GF, van Kemenade FJ, van Es LA, Bruijn JA, van Krieken JH. Endothelial cell chimerism after renal transplantation and vascular rejection. Lancet. 2001;357(9249):33-37.

28. Pober JS. Is host endothelium a silver lining for allografts? Lancet. 2001;357(9249):2-3.

29. Semenza GL. Oxygen homeostasis. Wiley Interdiscip Rev Syst Biol Med. 2010;2(3):336-361.

30. Nizet V,Johnson RS. Interdependence of hypoxic and innate immune responses. Nat Rev Immunol. 2009; 9(9):609-617.

31. Sitkovsky M, Lukashev D. Regulation of immune cells by local-tissue oxygen tension: HIF1 alpha and adenosine receptors. Nat Rev Immunol. 2005; 5(9):712-721.

32. Kobayashi $\mathrm{K}$, et al. A tissue-engineered trachea derived from a framed collagen scaffold, gingival fibroblasts and adipose-derived stem cells. Biomaterials. 2010;31(18):4855-4863.

33. Mazzone M, et al. Heterozygous deficiency of PHD2 restores tumor oxygenation and inhibits metastasis via endothelial normalization. Cell. 2009; 136(5):839-851.

34. Al-Lamki RS, Bradley JR, Pober JS. Endothelial cells in allograft rejection. Transplantation. 2008; 86(10):1340-1348.

35. Belperio JA, et al. Critical role for the chemokine MCP-1/CCR2 in the pathogenesis of bronchiolitis obliterans syndrome. J Clin Invest. 2001; 108(4):547-556.

36. Gasse P, et al. IL-1R1/MyD88 signaling and the inflammasome are essential in pulmonary inflammation and fibrosis in mice. J Clin Invest. 2007; 117(12):3786-3799.

37. Shilling RA, Wilkes DS. Immunobiology of chronic lung allograft dysfunction: new insights from the bench and beyond. Am J Transplant. 2009; 9(8):1714-1718.
38. Dacic S, Yousem SA. Histologic classification of idiopathic chronic interstitial pneumonias. Am J Respir Cell Mol Biol. 2003;29(3 suppl):S5-S9.

39. Phillips RJ, et al. Circulating fibrocytes traffic to the lungs in response to CXCL12 and mediate fibrosis. J Clin Invest. 2004;114(3):438-446.

40. Burlingham WJ, et al. IL-17-dependent cellular immunity to collagen type $\mathrm{V}$ predisposes to obliterative bronchiolitis in human lung transplants. J Clin Invest. 2007;117(11):3498-3506.

41. Iwano M, Neilson EG. Mechanisms of tubulointerstitial fibrosis. Curr Opin Nephrol Hypertens. 2004; 13(3):279-284.

42. Norman JT, Clark IM, Garcia PL. Hypoxia promotes fibrogenesis in human renal fibroblasts. Kidney Int. 2000;58(6):2351-2366.

43. Short M, Nemenoff RA, Zawada WM, Stenmark KR, Das M. Hypoxia induces differentiation of pulmonary artery adventitial fibroblasts into myofibroblasts. Am J Physiol Cell Physiol. 2004; 286(2):C416-C425

44. Du R, et al. HIF1alpha induces the recruitment of bone marrow-derived vascular modulatory cells to regulate tumor angiogenesis and invasion. Cancer Cell. 2008;13(3):206-220.

45. Grunewald M, et al. VEGF-induced adult neovascularization: recruitment, retention, and role of accessory cells. Cell. 2006;124(1):175-189.

46. Hattori $\mathrm{K}$, et al. Placental growth factor reconstitutes hematopoiesis by recruiting VEGFR1(+) stem cells from bone-marrow microenvironment. Nat Med. 2002;8(8):841-849.

47. Kioi M, Vogel H, Schultz G, Hoffman RM, Harsh GR, Brown JM. Inhibition of vasculogenesis, but not angiogenesis, prevents the recurrence of glioblastoma after irradiation in mice. J Clin Invest. 2010; 120(3):694-705.

48. Lin EY, et al. Macrophages regulate the angiogenic switch in a mouse model of breast cancer. Cancer Res. 2006;66(23):11238-11246

49. Yang L, et al. Expansion of myeloid immune suppressor $\mathrm{Gr}+\mathrm{CD} 11 \mathrm{~b}+$ cells in tumor-bearing host directly promotes tumor angiogenesis. Cancer Cell. 2004;6(4):409-421.

50. Carmeliet P. Mechanisms of angiogenesis and arteriogenesis. Nat Med. 2000;6(4):389-395

51. Nicolls MR, Zamora MR. Bronchial blood supply after lung transplantation without bronchial artery revascularization. Curr Opin Organ Transplant. 2010;15(5):563-567. 